\title{
Mesh objective modeling of cracks using continuous linear strain and displacement interpolations
}

\author{
M. Cervera*, ${ }^{*}$, M. Chiumenti and R. Codina \\ International Center for Numerical Methods in Engineering (CIMNE), Technical University of Catalonia (UPC), \\ Edificio C1, Campus Norte, Jordi Girona 1-3, 08034 Barcelona, Spain
}

\begin{abstract}
SUMMARY
The paper addresses the problem of tensile and mixed-mode cracking within the so-called smeared crack approach. Because lack of point-wise convergence on stresses is deemed as the main difficulty to be overcome in the discrete problem, a (stabilized) mixed formulation with continuous linear strain and displacement interpolations is used. The necessary convergence rate can be proved for such a formulation, at least in the linear problem. Two standard local isotropic Rankine damage models with strain-softening, differing in the definition of the damage criteria, are used as discrete constitutive model. Numerical examples demonstrate the application of the proposed formulation using linear triangular $P 1 P 1$ and bilinear quadrilateral $Q 1 Q 1$ mixed elements. The results obtained do not suffer from spurious mesh-bias dependence without the use of auxiliary tracking techniques. Copyright (C) 2011 John Wiley \& Sons, Ltd.
\end{abstract}

Received 21 September 2010; Revised 23 December 2010; Accepted 10 January 2011

KEY WORDS: mesh independence; tensile cracking; mixed-mode cracking; mixed finite elements; stabilization; local damage models

\section{INTRODUCTION}

Crack modeling has been one of the foci of interest in non-linear computational solid mechanics practically from its inception. The first record is the work of $\mathrm{Ngo}$ and Scordelis [1], who in 1967 studied a simply supported reinforced concrete beam and modeled crack propagation by the separation of nodal points using a tensile stress criterion. This can be considered as the seminal work of the discontinuous (or discrete) approach to crack modeling. In the discontinuous crack models, displacement jumps across the crack are explicitly considered and the non-linear behavior is established through a softening traction-displacement jump law.

Only months later, in 1968, Rashid [2] published a study of prestressed concrete pressure vessels modeling cracks using a crack model defined at constitutive level, also employing a tensile stress criterion for crack initiation. This was the origin of the continuous (or smeared) approach to crack modeling. In the continuous crack models, displacement jumps across the crack are smeared over the affected elements and the behavior of the crack is established through a softening stressstrain law.

The interest in cracks is only natural from the engineering point of view, because cracks are present to some degree in all structures. They may be present as basic defects in the constituent

\footnotetext{
${ }^{*}$ Correspondence to: M. Cervera, International Center for Numerical Methods in Engineering (CIMNE), Technical University of Catalonia (UPC), Edificio C1, Campus Norte, Jordi Girona 1-3, 08034 Barcelona, Spain.

†E-mail: miguel.cervera@upc.edu
} 
materials, or they may be induced by inadequate design or construction or during service life. Structural failure due to catastrophic crack propagation under inauspicious circumstances is a matter of concern of design and analysis in many fields of engineering.

Consequently, numerical simulation of tensile and mixed mode fracture has been the object of intensive interest during the last four decades. Both the discrete and the smeared crack approaches have been used and, through the years, they have come to have more points in common than originally thought. For instance, it is nowadays generally accepted that the amount of energy released during the formation of a unit area of crack surface is a material property, called the fracture energy. This important step forward was first implemented in a finite element analysis by Hillerborg et al. [3] in 1976, using the discrete approach; later on, Pietruszczak and Mróz [4] in 1981 and Bazant and Oh [5] in 1983 introduced the concept in the context of smeared models. Today, it is recognized that both proposals were equivalent, in the sense that they both sketched numerical approaches to solve (almost) the same continuum problem.

The fact of assuming that the strain energy in the structure is released through the crack surfaces has deen implications in the formulation of the problem, both at continuum and discrete level, that is, before and after proceeding to the spatial discretization into finite elements. At continuum level (before discretization), it explains what is called as structural size effect [6], and links the brittleness of the structural behavior to its size (larger size, more brittle behavior). At discrete level (after discretization), if using a smeared approach, it makes it necessary to link the computational local brittleness of the smeared crack to the resolution of the mesh and the element size (the wider the crack, more brittle the local behavior).

But regardless of many efforts devoted in the last 40 years to enhance the discrete and smeared crack approaches and to discuss their relative merits, the fact is that most attempts to model cracks

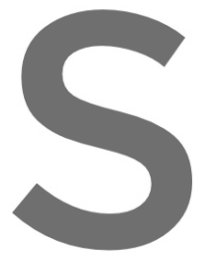
using standard, irred terms of collapse $n$ dependence in such A substantial part has been devoted among others) usually
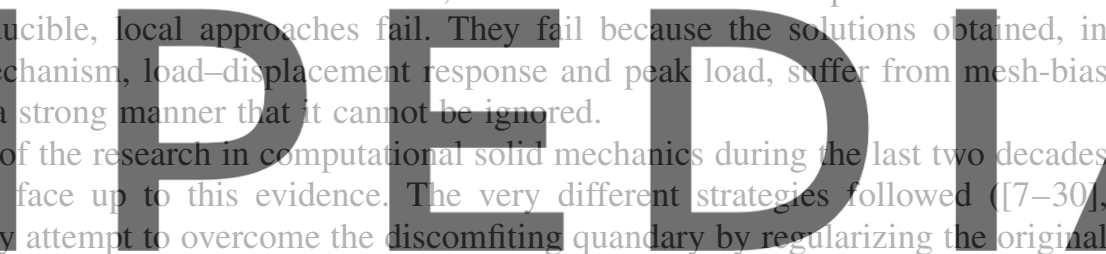
continuum problem, either introducing some additional length or time scale in it.

modeling. Our approach to tackle the problem is to resort from the standard irreducible formulation

to a mixed one, having displacements and strains as variables to be interpolated independently. The outcome of this is that improved accuracy and stability can be attained for the latter. This improvement can be crucial if strains are used to evaluate strongly non-linear constitutive laws, as in the case of crack modeling using the smeared approach. This is the core idea developed in this work.

Nevertheless, the use of mixed formulations is involved from the implementation point of view. The main reason for this is that the inf-sup compatibility condition poses severe restrictions on the selection of the pairs of discrete solution spaces. The alternative to use non-standard, exotic interpolations for these fields is to modify the discrete variational equations so as to allow for arbitrary interpolations, and, in particular, equal order for both fields. This is the essence of stabilized formulations.

In the last years, stabilized mixed displacement-pressure methods have been applied to the solution of $J_{2}$ elasto-plastic and damage problems [31-37]. Such a formulation produces a wellposed and numerically fully stable FE problem, even when strain softening behavior is considered, leading to the formation of slip lines. The results obtained, both in terms of collapse mechanism and global load-deflection response, are practically mesh independent. More recently, in [38, 39], a stabilized mixed strain-displacement method is formulated to tackle problems involving tensile cracking using Rankine-type damage criteria. Also in this case, a well-posed and numerically stable discrete problem is obtained, leading to the computation of crack paths, peak loads and global load-deflection responses which are practically mesh independent. 
The objectives of the present paper are three-fold: (a) to formulate a discrete model for crack propagation which is well posed and fully stable from the numerical point of view; the model is based on a mixed formulation using continuous linear interpolations both for the strain and displacement fields and a local isotropic Rankine damage model, (b) to show that this formulation is convergent, so that results obtained are not spuriously dependent on the finite element mesh used and that on mesh refinement it approaches the original continuum problem and (c) to show that the results obtained depend on the actual material model adopted. Compared with [38, 39], in this paper we focus our emphasis on the strain-displacement approach, we present a thorough description of the smeared crack approach within the mixed framework and we solve new problems, such as mixed-mode cracking situations, intended to demonstrate the objectives indicated.

The outline of the paper is as follows. In Section 2, a stabilized mixed strain/displacement formulation for the solution of non-linear solid mechanics problems is outlined. The continuum problem and the corresponding discrete formulation are introduced. Following the ideas in $[38,39]$, stabilization of the latter is achieved by considering a residual-based subscale on the strain field. Stability and convergence properties of the proposed formulation are discussed. Section 3 describes a smeared isotropic Rankine damage model. After recalling the basic features of the smeared approach, two alternative Rankine-type damage models are formulated. Finally, two selected numerical examples involving finite elements meshes of linear triangles and bilinear quadrilaterals are presented to assess the generality and robustness of the proposed formulation.
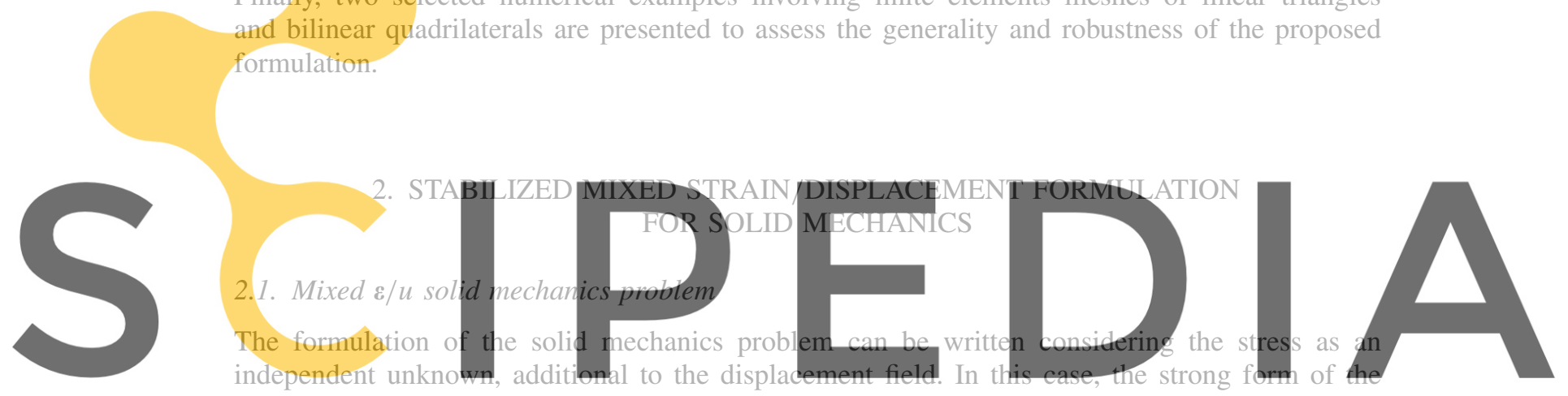

continuous problem can be stated as: given the prescribed body forces $\mathbf{f}$, find the displacement

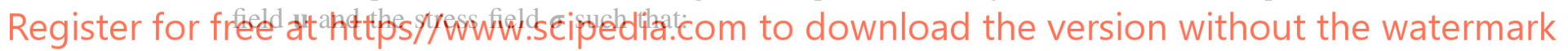

$$
\begin{array}{rr}
-\sigma+\mathrm{C}: \nabla^{s} \boldsymbol{u}=0 & \text { in } \Omega \\
\nabla \cdot \boldsymbol{\sigma}+\mathbf{f}=\mathbf{0} & \text { in } \Omega
\end{array}
$$

where $\Omega$ is the open and bounded domain of $\mathbb{R}^{n_{\mathrm{dim}}}$ occupied by the solid in a space of $n_{\mathrm{dim}}$ dimensions. Equation (1a) enforces the constitutive relationship, with $\mathbf{C}=\mathbf{C}(\boldsymbol{\sigma})$ being the non-linear constitutive tensor, while Equation ( $2 b)$ is the Cauchy equation.

Equations (1a)-(1b) are subjected to appropriate Dirichlet and Neumann boundary conditions. In the following, we will assume these, without loss of generality, in the form of prescribed displacements, $\mathbf{u}=\mathbf{0}$ on $\partial \boldsymbol{\Omega}_{u}$, and prescribed tractions, $\overline{\mathbf{t}}$ on $\partial \boldsymbol{\Omega}_{t}$, respectively, being $\partial \boldsymbol{\Omega}_{u}$ and $\partial \boldsymbol{\Omega}_{t}$ a partition of $\partial \Omega$.

This mixed formulation in terms of the stress and displacement fields, $\sigma / \mathbf{u}$, is classical and it has been used many times in the context of linear elasticity, where the constitutive tensor $\mathbf{C}$ is constant. However, it is not the most convenient format for the non-linear problem. The reason for this is that most of the algorithms used for non-linear constitutive equations in solid mechanics have been derived for the irreducible formulation. This means that these procedures are usually strain driven, and they have a format in which the stress $\sigma$ is computed in terms of the strain $\varepsilon$, with $\varepsilon=\nabla^{s} \mathbf{u}$. Because of this, it is advantageous to formulate the mixed solid mechanics problem considering the strain field $\varepsilon$, rather than the stress field $\sigma$, as an independent unknown, additional to the displacement field $\mathbf{u}$. 
In this case, the strong form of the continuum problem can be stated as: find the displacement field $\mathbf{u}$ and the strain field $\boldsymbol{\varepsilon}$, for given prescribed body forces $\mathbf{f}$ such that:

$$
\begin{array}{rr}
-\mathbf{C}: \boldsymbol{\varepsilon}+\mathbf{C}: \nabla^{s} \mathbf{u}=\mathbf{0} & \text { in } \Omega \\
\nabla \cdot(\mathbf{C}: \boldsymbol{\varepsilon})+\mathbf{f}=\mathbf{0} & \text { in } \Omega
\end{array}
$$

Equation (2a) enforces the non-linear constitutive relationship, with $\mathbf{C}=\mathbf{C}(\boldsymbol{\varepsilon})$ being the non-linear constitutive tensor, while Equation (2b) is the Cauchy equation. Equations (2a)-(2b) are also subjected to appropriate Dirichlet and Neumann boundary conditions of the same form as for (1a)-(1b).

Note that the strong form of the (standard) irreducible formulation, where the displacement field $\mathbf{u}$ is the only unknown, is obtained by the substitution of Equation (2a) into Equation (2b), to yield

$$
\nabla \cdot\left(\mathbf{C}: \nabla^{s} \mathbf{u}\right)+\mathbf{f}=\mathbf{0}
$$

Returning to the mixed formulation given by Equations (2a)-(2b), multiplying by the test functions and integrating by parts the second equation, the associated weak form of the mixed problem can be stated as

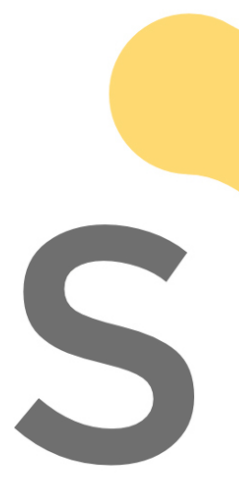

$$
\begin{aligned}
-(\boldsymbol{\gamma}, \mathbf{C}: \boldsymbol{\varepsilon})+\left(\gamma, \mathbf{C}: \nabla^{s} \mathbf{u}\right) & =0 \quad \forall \gamma \\
\left(\nabla^{s} \mathbf{v}, \mathbf{C}: \boldsymbol{\varepsilon}\right) & =(\mathbf{v}, \mathbf{f})+(\mathbf{v}, \overline{\mathbf{t}})_{\partial \Omega_{t}} \quad \forall \mathbf{v}
\end{aligned}
$$

where $\mathbf{v} \in \mathcal{V}$ and $\gamma \in \mathscr{G}$ are the variations of the displacements and strain fields, respectively, and $(\cdot, \cdot)$ denotes the inner orthogonality will be of the product of conditions of the class The definition of th to be only in $L^{2}(\Omega)$
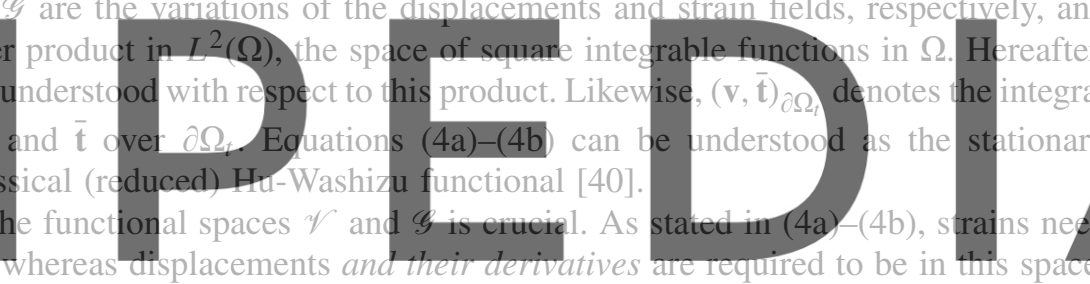

As for the Darcy problem (see $[41,42]$ ) this is the primal formulation of the problem. However,

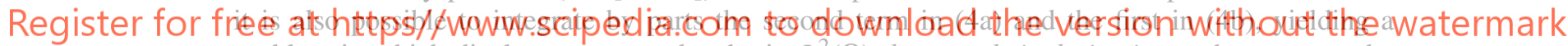
problem in which displacements need to be in $L^{2}(\Omega)$, but not their derivatives, whereas now the divergence of the strains needs to be in $L^{2}(\Omega)$. This corresponds to the dual formulation of the problem. Both possibilities, primal and dual, fit in our framework, and in fact the discrete problem presented next may be used in both cases. This is why we do not further specify the definition of $\mathscr{V}$ and $\mathscr{G}$.

The discrete Galerkin finite element counterpart problem is defined as:

$$
\begin{aligned}
-\left(\gamma_{h}, \mathbf{C}: \boldsymbol{\varepsilon}_{h}\right)+\left(\gamma_{h}, \mathbf{C}: \nabla^{s} \mathbf{u}_{h}\right) & =0 \quad \forall \gamma_{h} \\
\left(\nabla^{s} \mathbf{v}_{h}, \mathbf{C}: \boldsymbol{\varepsilon}_{h}\right) & =F\left(\mathbf{v}_{h}\right) \quad \forall \mathbf{v}_{h}
\end{aligned}
$$

where $\mathbf{u}_{h}, \mathbf{v}_{h} \in \mathscr{V}_{h}$ and $\boldsymbol{\varepsilon}_{h}, \gamma_{h} \in \mathscr{G}_{h}$ are the discrete displacement and strain fields and their variations, defined onto the finite element spaces $\mathscr{V}_{h}$ and $\mathscr{G}_{h}$, respectively, and $F\left(\mathbf{v}_{h}\right)=\left(\mathbf{v}_{h}, \mathbf{f}\right)+\left(\mathbf{v}_{h}, \overline{\mathbf{t}}\right)_{\partial \Omega_{t}}$. Note that the resulting system of equations is symmetric but non-definite.

The inf-sup condition $[43,44]$ establishes that the stability of the discrete formulation depends on appropriate compatibility restrictions on the choice of the finite element spaces $\mathscr{V}_{h}$ and $\mathscr{G}_{h}$, which we consider conforming and leave for the moment undefined. Standard Galerkin mixed elements with continuous equal order linear/linear interpolation for both fields do not satisfy the condition and, therefore, are not stable. For this particular problem, lack of stability manifests as spurious oscillations in the displacement field that entirely pollute the solution. A satisfactory way of eluding the constrictive inf-sup condition is to modify the discrete variational form, bringing into play appropriate numerical stabilization techniques that can provide the necessary stability. Such stabilization techniques can be satisfactorily sustained from the sub-grid scale approach [45]. 


\subsection{The sub-grid scale approach}

To apply the sub-grid scale approach to the continuum problem defined by Equations (4a)-(4b), the continuum strain field is split into two components, one coarse and a finer one, corresponding to different scales or levels of resolution. The solution of the continuum problem contains components from both scales. Consequently, for the solution of the discrete problem to be stable it is necessary to include the effect of both scales in the approximation. To this end, the strain field of the mixed problem is approximated as

$$
\boldsymbol{\varepsilon}=\boldsymbol{\varepsilon}_{h}+\widetilde{\boldsymbol{\varepsilon}}
$$

where $\boldsymbol{\varepsilon}_{h} \in \mathscr{G}_{h}$ is the strain component of the (coarse) finite element scale and $\widetilde{\boldsymbol{\varepsilon}} \in \widetilde{\mathscr{G}}$ is the enhancement of the strain field corresponding to the (finer) sub-grid scale. Let us also consider the corresponding variations $\gamma_{h} \in \mathscr{G}_{h}$ and $\widetilde{\gamma} \in \widetilde{\mathscr{G}}$, respectively. The strain solution space is $\mathscr{G} \simeq \mathscr{G}_{h} \oplus \widetilde{\mathscr{G}}$. The displacement field may also be split in the same manner, but this option will not be considered here for the sake of simplicity (even though it is needed to prove the results in [41]).

It is assumed that the coarse scale can be appropriately solved by a standard finite element interpolation, which however cannot solve the finer scale. Nevertheless, the effect of this finer scale needs to be included to enhance the stability of the displacement in the mixed formulation. The sub-grid strains $\widetilde{\boldsymbol{\varepsilon}}$ can be viewed as a perturbation of the finite element strain field $\varepsilon_{h}$, and it is reasonable to assume that they will be sufficiently 'small' compared with $\varepsilon_{h}$. It can also be assumed that $\widetilde{\boldsymbol{\varepsilon}}$ and $\tilde{\gamma}$ vanish on the boundary $\partial \Omega$.

Considering the subscales, the stresses, $\sigma=\mathrm{C}: \varepsilon$, can be decomposed into two different contributions
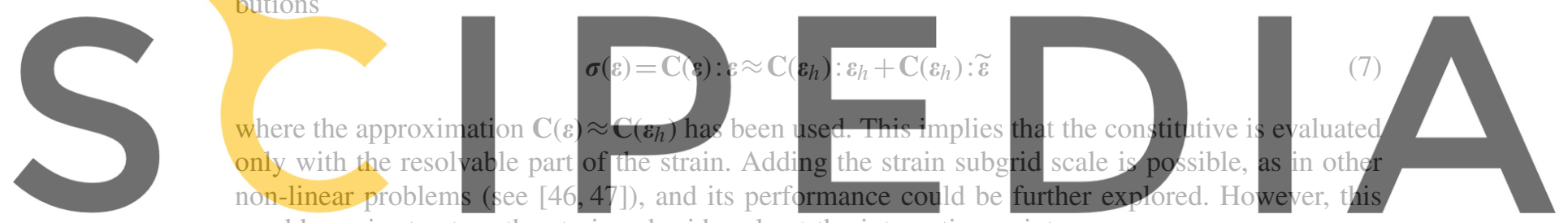

would require to store the strain subgrid scale at the integration points.

Considering the scale splitting, the discrete problem corresponding to Equations.(4a)-(4b) is:

$$
\begin{aligned}
-\left(\gamma_{h}, \mathbf{C}: \varepsilon_{h}\right)-\left(\gamma_{h}, \mathbf{C}: \widetilde{\varepsilon}\right)+\left(\gamma_{h}, \mathbf{C}: \nabla^{s} \mathbf{u}_{h}\right) & =0 \quad \forall \gamma_{h} \\
-\left(\widetilde{\gamma}, \mathbf{C}: \boldsymbol{\varepsilon}_{h}\right)-(\widetilde{\gamma}, \mathbf{C}: \widetilde{\boldsymbol{\varepsilon}})+\left(\widetilde{\gamma}, \mathbf{C}: \nabla^{s} \mathbf{u}_{h}\right) & =0 \quad \forall \widetilde{\gamma} \\
\left(\nabla^{s} \mathbf{v}_{h}, \mathbf{C}: \boldsymbol{\varepsilon}_{h}\right)+\left(\nabla^{s} \mathbf{v}_{h}, \mathbf{C}: \widetilde{\boldsymbol{\varepsilon}}\right) & =F\left(\mathbf{v}_{h}\right) \quad \forall \mathbf{v}_{h}
\end{aligned}
$$

Owing to the approximation used, Equation (6), and the linear independence of $\gamma_{h}$ and $\tilde{\gamma}$, the continuum Equation (4a) unfolds in two discrete equations, Equations (8a) and (8b), one related to each scale considered.

On one hand, Equations (8a) and (8c) are defined in the finite element spaces $\mathscr{G}_{h}$ and $\mathscr{V}_{h}$, respectively. The first one enforces the constitutive equation including a stabilization term $S_{1}=$ $\left(\gamma_{h}, \mathbf{C}: \widetilde{\boldsymbol{\varepsilon}}\right)$ depending on the sub-grid strains $\widetilde{\boldsymbol{\varepsilon}}$. The second one solves the balance of momentum including a stabilization term $S_{2}=\left(\nabla^{s} \mathbf{v}_{h}, \mathbf{C}: \widetilde{\boldsymbol{\varepsilon}}\right)$ depending on the sub-grid stresses $\widetilde{\boldsymbol{\sigma}}=\mathbf{C}: \widetilde{\boldsymbol{\varepsilon}}$.

On the other hand, Equation (8b) is defined in the sub-grid scale space $\widetilde{\mathscr{G}}$ and, hence, it cannot be solved by the finite element mesh. It can be rewritten as

$$
-(\widetilde{\gamma}, \mathbf{C}: \widetilde{\boldsymbol{\varepsilon}})=\left(\widetilde{\gamma}, \mathbf{r}_{h}\right) \quad \forall \widetilde{\gamma} \in \widetilde{\mathscr{G}}
$$

where the residual of the constitutive equation in the finite element scale is defined as:

$$
\mathbf{r}_{h}=\mathbf{r}_{h}\left(\boldsymbol{\varepsilon}_{h}, \mathbf{u}_{h}\right)=\mathbf{C}: \boldsymbol{\varepsilon}_{h}-\mathbf{C}: \nabla^{s} \mathbf{u}_{h}
$$


As the subgrid equation (9) cannot be solved by the finite element grid, to proceed it is necessary to provide an approximate closed-form solution to it. In addition, the format of Equation (9), which is exact for the fine scale and nonlocal, strongly suggests that $\mathbf{C}: \widetilde{\boldsymbol{\varepsilon}}$, and hence $\widetilde{\boldsymbol{\varepsilon}}$, are driven by the residual of the coarse scale, $\mathbf{r}_{h}$. In [48] it is reasoned that the sought effect of the finer scale is to explicitly account for the distributional effects of the residual of the coarse scale. Therefore, the residual-based subscale strain can be localized within each finite element $\Omega_{e}$, and be expressed as

$$
\widetilde{\boldsymbol{\varepsilon}}=\tau_{\varepsilon} \mathbf{C}^{-1}: \mathbf{r}_{h}=\tau_{\varepsilon}\left[\nabla^{s} \mathbf{u}_{h}-\boldsymbol{\varepsilon}_{h}\right]
$$

where $\tau_{\varepsilon}$ is computed as

$$
\tau_{\varepsilon}=c_{\varepsilon} \frac{h}{L}
$$

and where $c_{\varepsilon}$ is an algorithmic constant, $h$ is the element size and $L$ is a characteristic length of the computational domain. Equation (11) has to be understood as the approximate projection of $\nabla^{s} \mathbf{u}_{h}-\varepsilon_{h}$ onto the space of strain subscales, and therefore $\tau_{\varepsilon}$ is not necessarily 1 . The expression given by (12) has been chosen according to the optimal convergence results obtained for equal interpolation in [41], since in the following we precisely assume equal continuous interpolation for displacements and strains. Let us also mention that the projection orthogonal to the finite element space could be taken of the right-hand side of (11), although it turns out that the resulting scheme for this particular problem would be the same (see [38]).

Note that with the definition in Equation (11), the subscale $\widetilde{\varepsilon}$ is computed in an element by

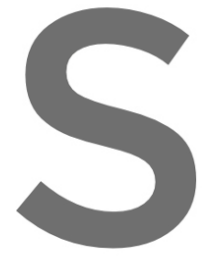

element manner and the discontinuous tinuous across ele even if defined eler continuous.

Introducing the can be written as
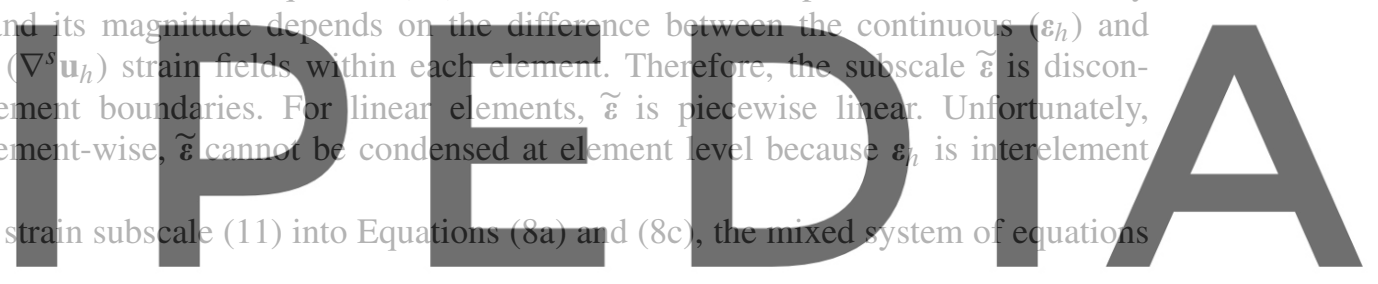

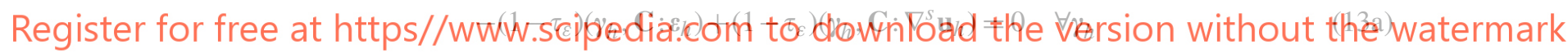

$$
\left(1-\tau_{\varepsilon}\right)\left(\nabla^{s} \mathbf{v}_{h}, \mathbf{C}: \varepsilon_{h}\right)+\tau_{\varepsilon}\left(\nabla^{s} \mathbf{v}_{h}, \mathbf{C}: \nabla^{s} \mathbf{u}_{h}\right)=F\left(\mathbf{v}_{h}\right) \quad \forall \mathbf{v}_{h}
$$

where the terms depending on $\tau_{\varepsilon}$ represent the stabilization. Note that the resulting system of equations is symmetric.

If $P_{C}$ is the $L^{2}(\Omega)$ projection weighted by $\mathbf{C}$, the projection involved in (13a) can be written as

$$
\boldsymbol{\varepsilon}_{h}=P_{C}\left(\nabla^{s} \mathbf{u}_{h}\right)
$$

and, therefore, the proposed stabilized mixed strain-displacement approach can also be written as:

$$
\begin{aligned}
\boldsymbol{\varepsilon}_{\mathrm{stab}} & =\left(1-\tau_{\varepsilon}\right) P_{C}\left(\nabla^{s} \mathbf{u}_{h}\right)+\tau_{\varepsilon}\left(\nabla^{s} \mathbf{u}_{h}\right) \\
\left(\nabla^{s} \mathbf{v}_{h}, \mathbf{C}: \boldsymbol{\varepsilon}_{\mathrm{stab}}\right) & =F\left(\mathbf{v}_{h}\right)
\end{aligned}
$$

It is noteworthy that the term added to secure a stable solution decreases rapidly upon mesh refinement, as the finite element scale becomes finer and the residual in Equation (10) reduces $\left(\widetilde{\varepsilon}\right.$ is sufficiently 'small' compared with $\boldsymbol{\varepsilon}_{h}$ ).

\subsection{Stability and convergence results}

Stability and convergence results for the stabilized mixed method given by (13a)-(13b) applied to the linear elastic problem, with $\mathbf{C}=\mathbf{C}_{0}$, have been collected in Table I, indicating only the order of convergence. This order is compared with what would be obtained with the irreducible 
Table I. Order of convergence of different terms in the irreducible and mixed stabilized formulations when interpolations of degree $k$ are used for both $\mathbf{u}_{h}$ and $\epsilon_{h}$.

\begin{tabular}{lcc}
\hline Term & Irreducible & Mixed \\
\hline$\left\|\nabla^{s}\left(\mathbf{u}-\mathbf{u}_{h}\right)\right\|$ & $h^{k}$ & $h^{k}$ \\
$\left\|\mathbf{u}-\mathbf{u}_{h}\right\|$ & $h^{k+1}$ with duality & $h^{k+1 / 2}$ without duality \\
$\left\|\boldsymbol{\epsilon}-\boldsymbol{\epsilon}_{h}\right\|$ & $h^{k}$ & $h^{k+1}$ with duality \\
& $\boldsymbol{\sigma}_{h}=\mathbf{C}_{0}: \nabla^{s} \mathbf{u}_{h}$ & $h^{k+1}$ without duality \\
& & $\boldsymbol{\sigma}_{h}=\mathbf{C}_{0}: \boldsymbol{\epsilon}_{h}$ \\
\hline
\end{tabular}

formulation, where the differential equation to be solved is Equation (3). The proof of these results can be done adapting the analysis presented in [41].

Table I shows that in the linear case the strains and stresses are approximated with a better accuracy using the stabilized mixed formulation. In this table, it is indicated whether a convergence result requires the typical elliptic regularity assumption used in duality arguments or not (see [41] for details). Note that, even if it does not hold, improved convergence in stresses can be achieved. As it has been already mentioned, in this work we are interested in non-linear constitutive behavior of materials of the secant form

$$
\mathrm{C}=\mathrm{C}(\varepsilon)
$$
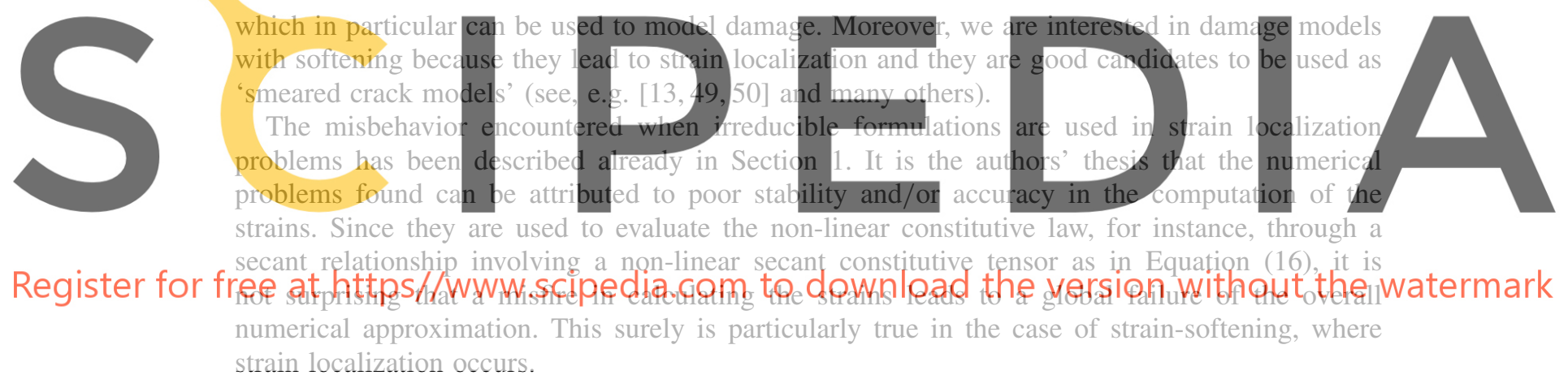

strain localization occurs.

Even though we do not have the analysis for non-linear problems, the results in Table I suggest that the stabilized mixed formulation presented may succeed in overcoming the numerical problems which are behind the observed misbehavior of the irreducible formulation. This is so mainly because strain and stress accuracy is improved, as the mixed formulation achieves better convergence rate on the strains (or stresses) than the irreducible formulation. This could not be considered a discriminating argument, as this improvement is attained at the cost of using more degrees of freedom for the same number of nodes in the FE mesh.

However, the real problem with the irreducible formulation is not a poor convergence rate on the strains, but lack of convergence. The norms evaluated in the mentioned Table I are global. Without additional regularity conditions, local estimates of convergence are expected to be one order smaller. This means that, using linear elements, $k=1$, no convergence order can be guaranteed for the strains that are used to evaluate the constitutive law (16) pointwise in the irreducible formulation. Propitiously, for the mixed stabilized formulation we can formally expect order $h$ convergence in the worst situation (order $h^{1 / 2}$ if the assumptions of duality arguments do not apply). Using higher order elements, $k>1$, in problems involving strong gradients and/or discontinuities does not improve the convergence estimates, since higher order derivatives involved in these estimates are not bounded in such situations. In fact, if the continuous solution is singular, not even the first order derivatives are going to be point-wise bounded. Nevertheless, convergence (without order) can be expected in the mixed formulation in the energy norm defined for a region around the singularity. This is not the case for the irreducible formulation. 


\section{SMEARED ISOTROPIC RANKINE DAMAGE MODELS}

\subsection{The smeared crack approach}

In this section a brief recollection of the smeared approach to crack modeling is made. Distinction between the formulation of the model at continuum level and its discrete counterpart, the FE implementation, is emphasized. It is shown that the smeared crack model offers different alternatives within the irreducible or mixed frameworks discussed in Section 2.

Consider the body occupying the domain $\Omega$, as shown in Figure 1(a), crossed by a discontinuity $S$, which represents a crack. Regions $\Omega^{+}$and $\Omega^{-}$are the parts of the body located 'in front' and 'behind' the crack. Let $L$ be a characteristic length of the domain.

At the continuum level, a crack model such as the one represented in Figure 2(a) may be adopted. The top graph shows the normal displacement along a line normal to the crack; it is
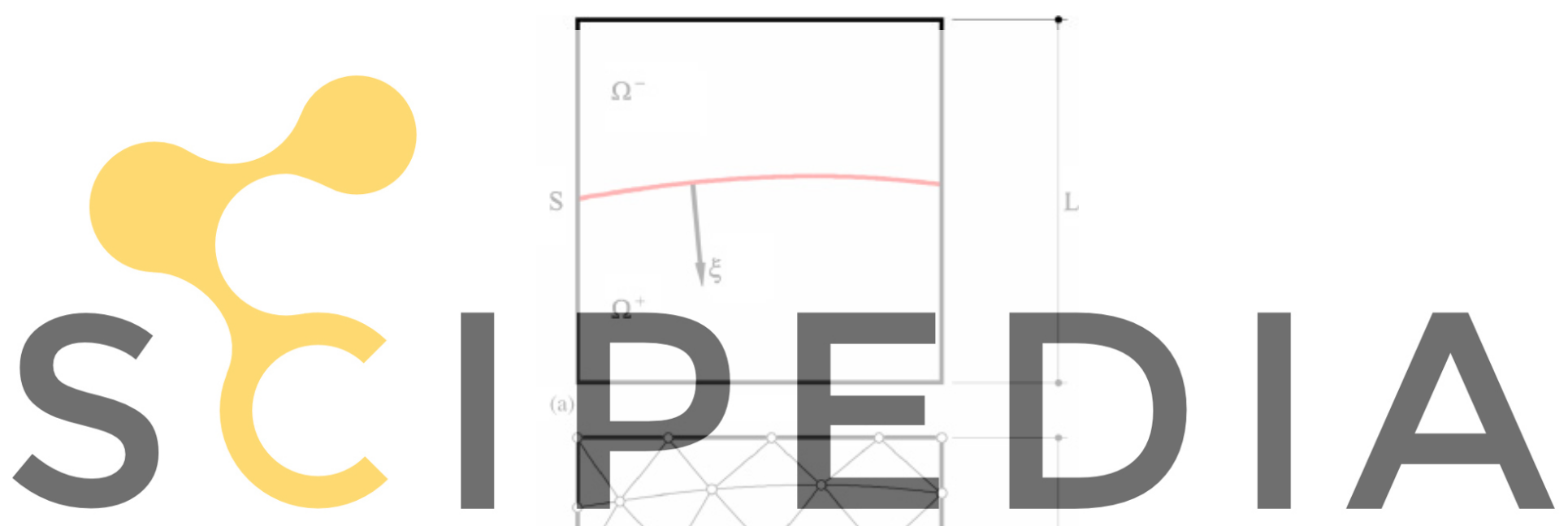

Register for free at https//www.Scipedia.com/to download the version without the watermark

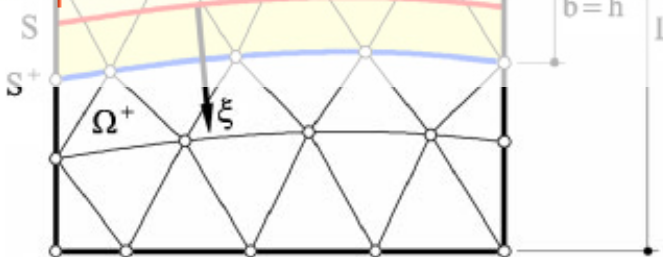

(b)

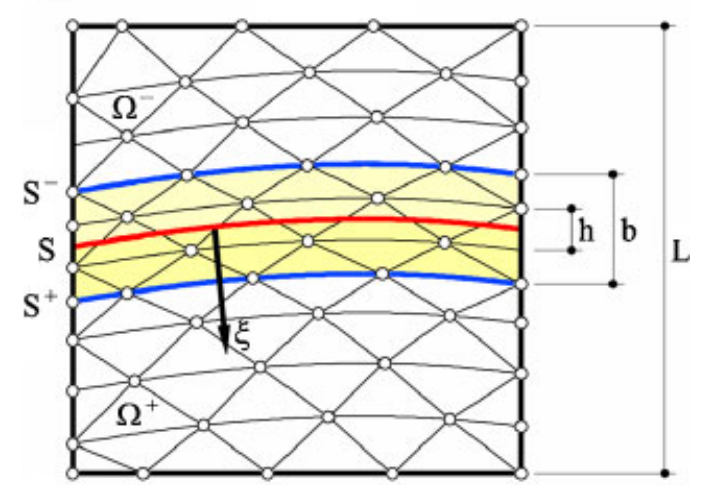

(c)

Figure 1. Crack models: (a) continuum model; (b) discrete-irreducible smeared model; and (c) discrete-mixed smeared model. 

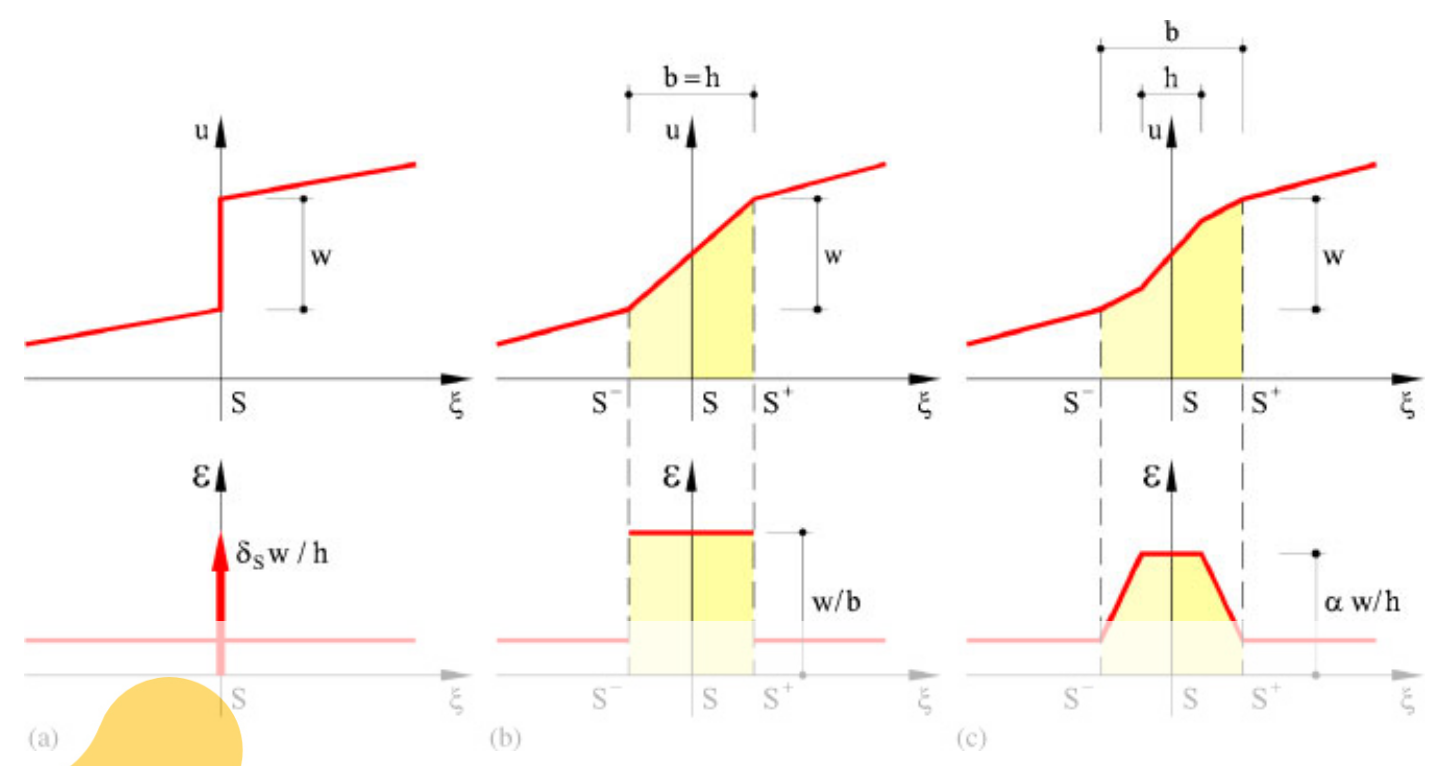

Figure 2. Displacements and strains in crack models: (a) continuum model; (b) discrete-irreducible smeared model; and (c) discrete-mixed smeared model.
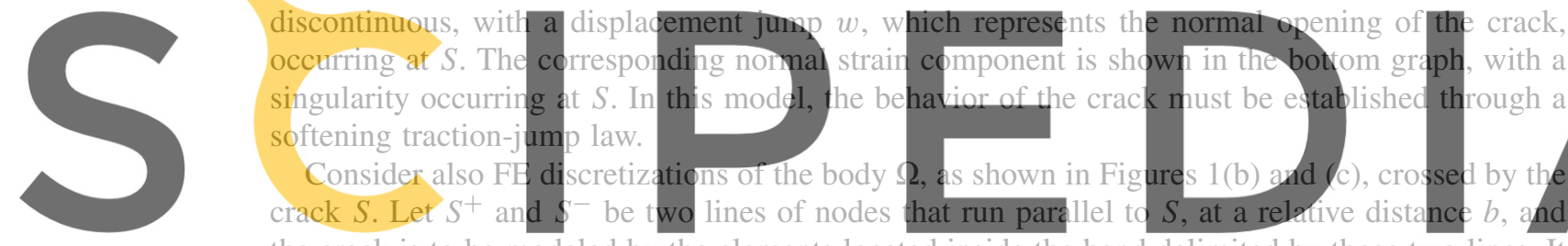

the crack is to be modeled by the elements located inside the band delimited by those two lines. It is stressed that the bandwidth $b$ is not a material parameter and it does not belong to the definjtion

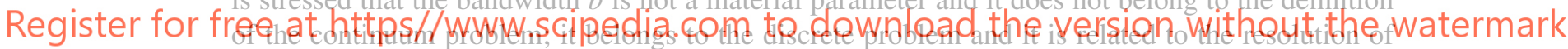

the mesh and the element size $h$. The ratio $\beta=b / h$ depends on the localization sharpness that a given FE formulation is able to achieve.

At the discrete level, a smeared crack model such as the one represented in Figure 2(b) may be considered. It corresponds to the standard smeared crack approach, based on the irreducible formulation of the mechanical problem, where the strain field is obtained by differentiation of the displacement field. Note that the displacement field is inter-element continuous, while the strain field is inter-element discontinuous. Because of this, for a given discretization, optimal resolution of the crack implies that $b=h, \beta=1$, that is, that the strain localization band is only one element across. As the behavior of the crack is established through a softening stress-(total) strain law, this type of model can be implemented in any non-linear FE code by simply writing a routine for a new material constitutive model.

Alternatively, it is possible to adopt a smeared crack model such as the one represented in Figure 2(c). It corresponds to the same smeared crack approach, but now based on the mixed strain/displacement formulation of the mechanical problem presented in Section 2, where both the strain and the displacement fields are independent variables. Note that here both fields are linear and inter-element continuous, since this is the situation we are interested in (see [42] for other alternatives). Because of this, for a given discretization, optimal resolution of the crack implies that $b>h, \beta>1$, that is, that strain localization affects more than one element across the crack. As in the standard model, the behavior of the crack is established through a softening stress-(total) strain law.

It is noteworthy that the basic idea behind the smeared approach to crack modeling is that, on mesh refinement, as the ratio $h / L$ decreases, both the strain and displacement fields obtained from 
the discrete (smeared) approach must: (a) converge and (b) converge to those of the continuum model.

Requirement (a) has been discussed in the last part of Section 2. There is no guarantee of point-wise convergence of the strains and stresses for the standard irreducible FE formulation in the general case. This unfortunate circumstance is responsible for the often observed lack of objectivity of the results obtained with irreducible formulations with regard to mesh alignment. It should be noted that this drawback is not due to the smeared approach to crack modeling. It is due to the limitations of the irreducible formulation and, thus, it occurs also in approaches incorporating discontinuous displacement interpolations, either via element or nodal enrichments.

Requirement (b) needs energy dissipation during the crack propagation to be considered, both at the continuum and discrete levels. This was first done by Pietruszczak and Mróz in 1981 [4] and Bazant and Oh in 1983 [5] and it is done routinely in smeared crack models ever since [51-55].

On one hand, let us assume that the continuum non-linear problem is defined by the following material properties: the elastic modulus $E$, the cracking strength $\sigma_{0}$ and the fracture energy $G$, defined as the energy dissipated per unit area when a fracture surface forms. From the first two properties, the elastic strain energy stored per unit volume when a fracture surface forms is $\mathscr{U}_{0}=\sigma_{0}^{2} / 2 E$. The three material properties can be combined to define the material (characteristic) length, $\mathscr{L}=2 E G / \sigma_{0}^{2}$.

Recalling that $L$ is a characteristic length of the domain, the ratio between the stored elastic energy and the released fracture energy is a dimensionless parameter:

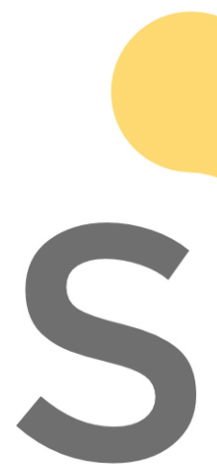

$$
\Pi^{B}=\frac{\varkappa_{0} L}{G}=\frac{L}{\mathscr{L}}
$$

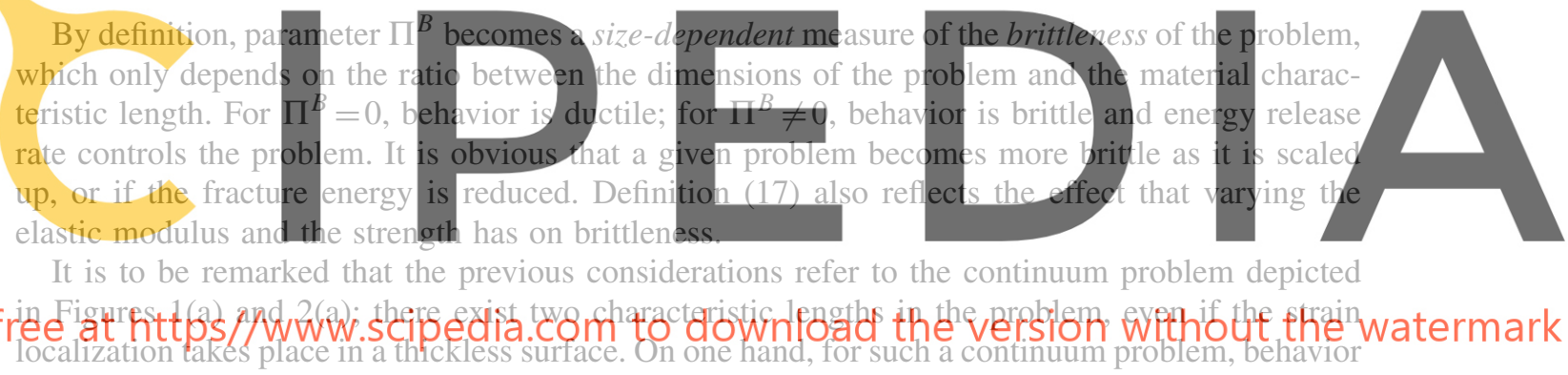

of the crack is established through a softening traction-(displacement) jump law, which can be established in terms of $\mathscr{L}$ or, in a non-dimensional fashion, in terms of $\Pi^{B}$. It is ciear that such a setting incorporates the structural size-effect in a straightforward manner [37].

On the other hand, in a discrete smeared model, behavior of the crack is established through a softening stress-(total) strain law, and the phenomena of cracking and fracture are modeled via strain localization. Let us assume that the strain localization band has a finite bandwidth $b$, inside which the discrete energy dissipation $\mathscr{D}$, defined per unit volume, takes place. In this case, the brittleness parameter of the discrete problem can be written as:

$$
\Pi_{\mathrm{S}}^{B}=\frac{\mathscr{U}_{0} L}{\mathscr{D} b}=\frac{L}{\mathscr{L}}\left(\frac{G}{\mathscr{D} b}\right)
$$

From this expression, it is clear that consistency between the discrete smeared model and the continuum problem requires that the equality

$$
\mathscr{D} b=G
$$

holds in the discrete model. For the discrete model, if Equation (19) holds, Equation (18) may also be rewritten as:

$$
\Pi_{\mathrm{S}}^{B}=\frac{L}{\mathscr{L}}=\frac{L}{b} \pi^{B}
$$

The ratio $L / b$ defines the resolution (sharpness) of the localization band related to the problem size, while the new non-dimensional number $\pi^{B}=b / \mathscr{L}$ defines the brittleness inside the band. 
This implies that the discrete softening stress-strain law must be established, in a non-dimensional fashion, in terms of $\pi^{B}$. Note that, on mesh refinement, as $b$ reduces, $\pi^{B}$ reduces in the same measure, and the brittleness in the band reduces accordingly.

\subsection{Isotropic Rankine damage model}

The constitutive equation for the scalar isotropic damage model used in this work is:

$$
\boldsymbol{\sigma}=\mathbf{C}: \boldsymbol{\varepsilon}=(1-d) \mathbf{C}_{0}: \boldsymbol{\varepsilon}
$$

where the stresses $\boldsymbol{\sigma}$ can be computed in terms of the total strain tensor $\boldsymbol{\varepsilon}$, the linear elastic constitutive tensor $\mathbf{C}_{0}$ and the damage index $d$.

The damage model is completed with the definition of the evolution of the damage index in terms of the evolution of the total strains, or the effective stresses $\overline{\boldsymbol{\sigma}}$, defined as $\overline{\boldsymbol{\sigma}}=\mathbf{C}_{0}: \boldsymbol{\varepsilon}$.

To model tensile damage, the equivalent effective stress, $\tau$, is defined in one of the following manners:

$$
\begin{aligned}
\tau & =\left\langle\bar{\sigma}_{1}\right\rangle \\
\tau & =\sum_{i=1}^{3}\left\langle\bar{\sigma}_{i}\right\rangle
\end{aligned}
$$

where $\bar{\sigma}_{i}, i=1,2,3$, are the principal effective stresses, $\bar{\sigma}_{1}$ being the largest one and $\langle\cdot\rangle$ are the Macaulay brackets $(\langle x\rangle=x$, if $x \geqslant 0,\langle x\rangle=0$, if $x<0)$.

With one of these definitions for the equivalent effective stress, the damage criterion, $\Phi$, is introduced as

$$
\Phi(\tau, q)=\tau-r \leqslant 0
$$

where variable $r$ is the current damage threshold, an internal stress-like variable whose value controls the size of the damage surface. The initial value of the damage threshold is $r_{0}=\sigma_{0}$, where $\sigma_{0}$ is the initial uniaxial damage stress, that is, the tensile strength.

Together with Equation (22a), Equation (23) corresponds to the standard Rankine criterion, whereas together with Equation (22b), it corresponds to the truncated Rankine criterion, sensitive to multiaxial tension states. Figure 3(a) shows a schematic representation of the defined damage criteria on the $\sigma_{3}=0$ plane of the stress space.

The evolution of the damage bounding surface for loading, unloading and reloading conditions is controlled by the Kuhn-Tucker relations and the damage consistency condition, which are

$$
\begin{gathered}
\dot{r} \geqslant 0 \quad \Phi(\tau, r) \leqslant 0 \quad \dot{r} \Phi(\tau, r)=0 \\
\text { if } \Phi(\tau, r)=0 \quad \text { then } \dot{r} \dot{\Phi}(\tau, r)=0
\end{gathered}
$$
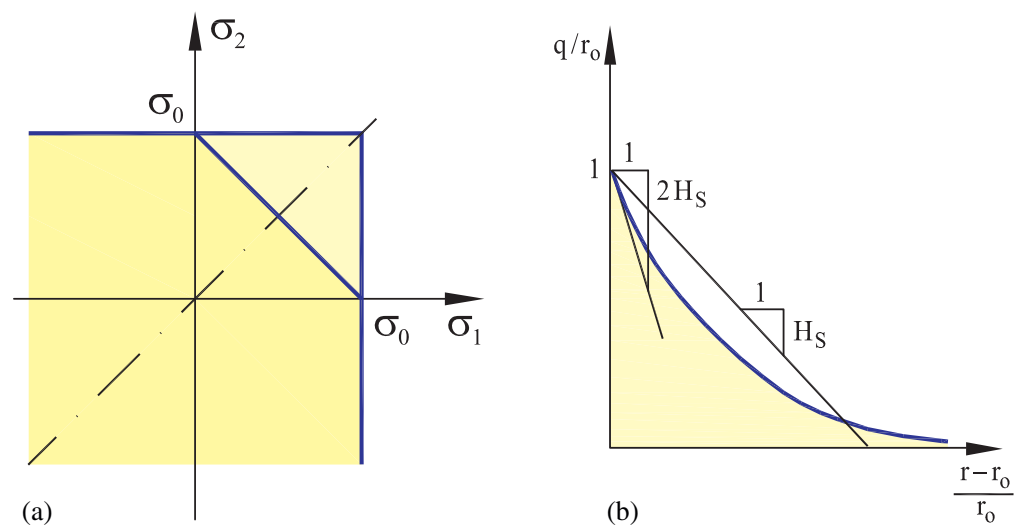

Figure 3. Rankine damage model: (a) damage surfaces and (b) softening function. 
leading, in view of Equation (23), to the loading condition

$$
\dot{r}=\dot{\tau}
$$

This, in turn, leads to the explicit definition of the current values of the internal variable $r$ in the form

$$
r=\max \left(r_{0}, \max _{t \in[0, \infty)} \tau(t)\right)
$$

Stress softening is controlled by the softening function $q=q(r)$. In this work, the following exponential softening law is used:

$$
q(r)=r_{0} \exp \left(-2 H_{\mathrm{S}}\left(\frac{r-r_{0}}{r_{0}}\right)\right), \quad r \geqslant r_{0}
$$

where $H_{\mathrm{S}} \geqslant 0$ is the softening parameter. Figure $3(\mathrm{~b})$ shows a schematic representation of this function.

Finally, the damage index $d=d(r)$ is explicitly defined in terms of the corresponding current value of the damage threshold

$$
d(r)=1-\frac{q(r)}{r}, \quad r \geqslant r_{0}
$$

so that it is a monotonically increasing function such that $0 \leqslant d \leqslant 1$.

\subsection{Mechanical dissipation}

The mechanical free energy for an isotropic damage model is defined as:

$$
W=(1-d) W^{e}(\boldsymbol{\varepsilon})=(1-d) \frac{1}{2}\left[\boldsymbol{\varepsilon}: \mathbf{C}_{0}: \boldsymbol{\varepsilon}\right] \geqslant 0
$$

Thus, the rate of mechanical dissipation can be expressed as:

$$
\dot{\mathscr{D}}=W^{e} \dot{d} \geqslant 0
$$

Let us consider an uniaxial tensile experiment in which the tensile strain $\varepsilon$ increases monotonically and quasi-statically from an initial unstressed state to another in which full degradation takes place. In this case, $W^{e}=E \varepsilon^{2} / 2=\bar{\sigma}^{2} / 2 E=r^{2} / 2 E$, where $E$ is Young's modulus and $r=\bar{\sigma}=E \varepsilon$.

Using Equations (30) and (28), the specific energy dissipated in the process is:

$$
\begin{aligned}
\mathscr{D} & =\int_{0}^{\infty} \dot{\mathscr{D}} d t=\int_{0}^{\infty} W^{e} \dot{d} d t \\
& =\frac{1}{2 E}\left(\int_{r_{0}}^{\infty} q d r-\int_{q_{0}}^{0} r d q\right)=\frac{1}{2 E}\left(r_{0} q_{0}+2 \int_{r_{0}}^{\infty} q d r\right)
\end{aligned}
$$

where $\dot{d} d t=d^{\prime} d r=\left(q-r q^{\prime}\right) d r / r^{2}=(q d r-r d q) / r^{2}$ and $r_{0}=\sigma_{0}, q_{0}=q\left(r_{0}\right)=\sigma_{0}$. Using Equation (27), it results

$$
\mathscr{D}=\left(1+\frac{1}{H_{S}}\right) \frac{\sigma_{0}^{2}}{2 E}=\left(1+\frac{1}{H_{S}}\right) \mathscr{U}_{0}
$$

Recalling the considerations in Section 3.1 and, in particular, the fact that Equation (19) must hold for the discrete constitutive model, it is

$$
H_{S}=\frac{b}{\mathscr{L}-b}=\frac{\pi^{B}}{1-\pi^{B}}
$$

where $b$ is the width of the localization band, $\mathscr{L}=2 E G / \sigma_{0}^{2}$ is the material length and $\pi^{B}=b / \mathscr{L}$. Equations (27) and (33) establish that softening in the discrete smeared model depends only on $\pi^{B}$, as stated in Section 3.1. 
In quasi-static conditions, $\mathscr{D}$ must be greater than $\mathscr{U}_{0}$, and this requires that $H_{\mathrm{S}}$ must be positive, so that $b \leqslant \mathscr{L}\left(\pi^{B} \leqslant 1\right)$. Recalling that $b$ is related to the element size $h, \beta=b / h$, this condition sets a maximum size for the elements that can be used in the analysis, $h \leqslant \mathscr{L} / \beta$.

For linear elements and in the irreducible formulation, the discrete localization band is only one element across, and $\beta=1$ [56]. For the mixed problem, inter-elemental strain continuity implies an effective discrete localization bandwidth with $\beta=2$, see [39]. In this work, the size of the element is computed as $h^{2}=2 A$ for triangular elements and $h^{2}=A$ for quadrilateral elements, where $A$ is the area of the element.

\section{NUMERICAL EXAMPLES}

The application of the stabilized mixed strain/displacement $\boldsymbol{\varepsilon} / \mathbf{u}$ formulation presented in this work to the problem of tensile cracking and associated strain localization is illustrated below in two different demonstrative examples. Relative performance of the irreducible displacement formulation and the stabilized mixed strain/displacement formulation is tested considering 2D 3-node triangular and 4-node quadrilateral meshes. The elements used will be triangular $P 1$ (linear displacement) and $P 1 P 1$ (linear strain/linear displacement) elements preferentially, but the formulation presented is very general and results comparing quadrilateral $Q 1$ (bilinear displacement), $Q 1 Q 1$ (bilinear strain/bilinear displacement) elements are also shown. Only low-order elements are considered because they are more effective in problems involving sharp displacement and strain gradients. When the stabilized mixed strain/displacement formulation is used, a value of $c_{\varepsilon}=1.0$ is used for the evaluation of the stabilization parameters $\tau_{\varepsilon}$. In both examples, the height of the beams is taken as representative length of the problem, $L$. Tensile cracking is modeled using the local scalar damage model with exponential strain softening described in Section 3.

The discrete finite element problem is solved incrementally, in a (pseudo)-time step-by-step manner. Analyses are performed under displacement control in order to trace the complete postpeak behavior. An automatic time incrementation procedure is used to reduce the size of the time steps when convergence due to the non-linear effects is more difficult. Within each step, convergence is attained when the ratio between the norm of the iterative and the incremental norm of the residual arrays is lower than $10^{-3}$. It has to be remarked that no tracking algorithm of any sort has been used in any of the computations.

Calculations are performed with an enhanced version of the finite element program COMET [57], developed at the International Center for Numerical Methods in Engineering (CIMNE). Pre and post-processing is done with $\mathrm{GiD}$, also developed at CIMNE [58].

No discussion about the relative cost of the present approach compared with the irreducible one will be presented in the following. Comparison tests are reported in [38], where it is concluded that the increase is affordable. Nevertheless, research aiming to improve the performance of the mixed formulation will be pursued elsewhere.

\subsection{Four-point bending beam}

The first example is a plane-strain doubly notched beam subjected to four point bending. Figure 4 depicts the geometry of the problem; dimensions of the beam are $134.0 \mathrm{~cm} \times 30.6 \mathrm{~cm}$ (width $\times$ height) and the length and width of the notches are 8.2 and $0.5 \mathrm{~cm}$, respectively. The load is applied at the central (rigid) supports (at $8.0 \mathrm{~cm}$ from the center of the beam) by imposing vertical displacements of opposite sign at the top and bottom supports. The two supports near the extremes of the beam (at $20.3 \mathrm{~cm}$ ) are fixed. This example is selected because it represents an excellent example of mixed mode fracture. It presents two singular points at the tips of the notches; tensile stresses are very large in the vicinity of these regions and damage starts there.

The following material properties are assumed: Young's modulus $E=30 \mathrm{GPa}$, the Poisson ratio $v=0.2$, tensile strength $\sigma_{0}=2 \mathrm{MPa}$ and mode I fracture energy $G=100 \mathrm{~J} / \mathrm{m}^{2}$. Both the standard and the truncated Rankine damage criteria defined in Figure 3(a) are used. 


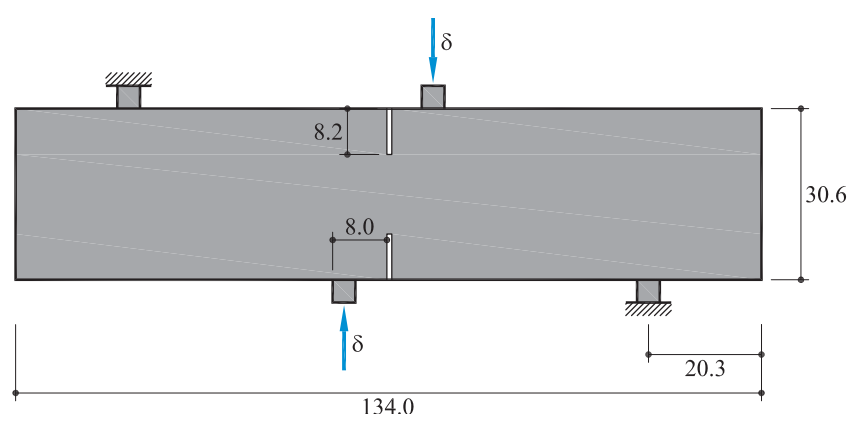

Figure 4. Geometry and load for four-point bending beam.

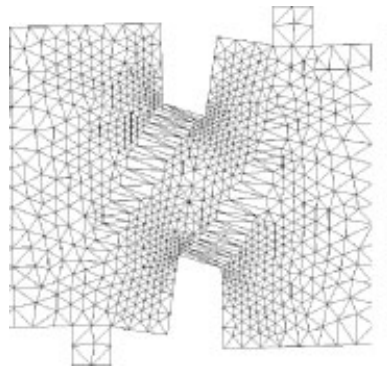

(a1)

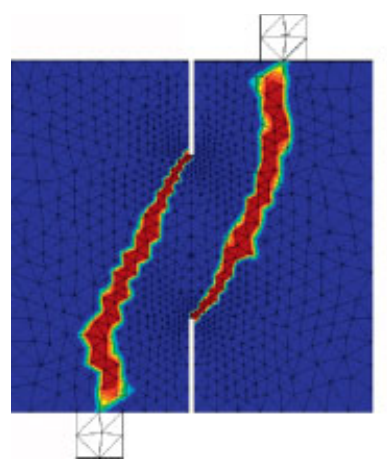

(a2)

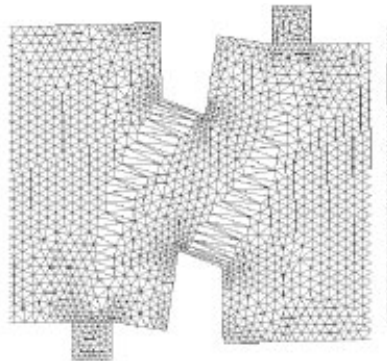

(b1)

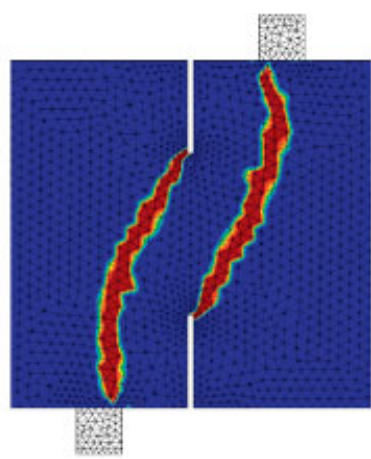

(b2)

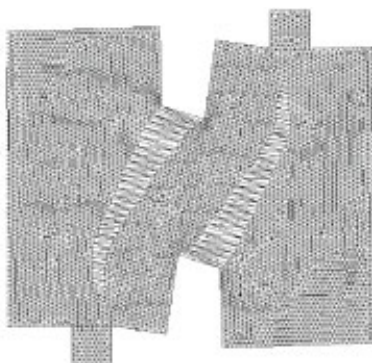

(c1)

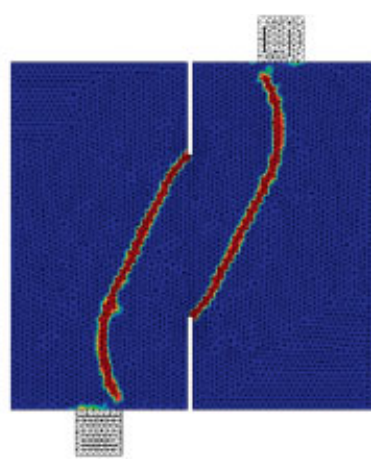

(c2)

Figure 5. Deformed geometries $(\times 100)$ and damage index contours on the three meshes with mixed $P 1 P 1$ elements (standard Rankine criterion).

The computational domain is discretized in three different unstructured meshes with average mesh sizes in the central part of (a) $h_{e}=20 \mathrm{~mm}$ (1189 nodes), (b) $h_{e}=10 \mathrm{~mm}$ (1959 nodes) and (c) $h_{e}=5 \mathrm{~mm}$ (5909 nodes). The central part of the three meshes is shown in Figures 5 and 6.

First, $P 1 P 1$ (linear strain/linear displacement) mixed elements are used. Three separate analyses are performed using these meshes and the standard Rankine damage criterion (see Equations (22a) and (23) and Figure 3(a)). The computed deformed shapes of the central part of the beam are shown in Figures 5(a.1), (b.1) and (c.1), respectively (imposed vertical displacement $\delta=0.1 \mathrm{~mm}$, with a displacement amplification factor of 100). The different element sizes in the meshes can be appreciated in these figures. As shown, the computed cracks in all the analyses follow very closely the same path, starting at the tip of the notches and progressing, mostly parallel to each other. Note that these two cracks are fairly straight and, in practical terms, they define the limits of the compressive strut that links the two central supports. They turn vertically when they reach the action lines of the reactions of the central supports. The computed crack pattern corresponds 


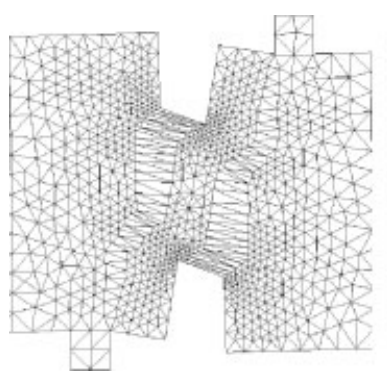

(a1)

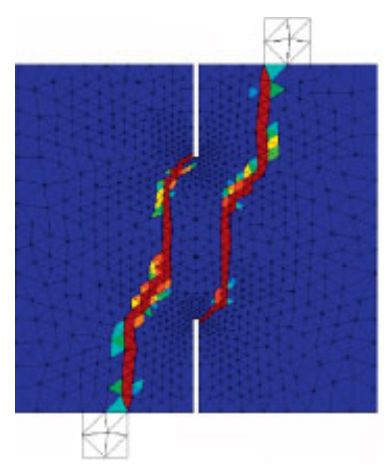

(a2)

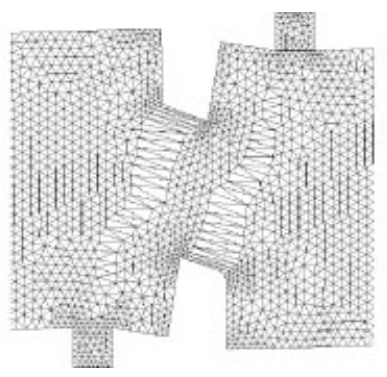

(b1)

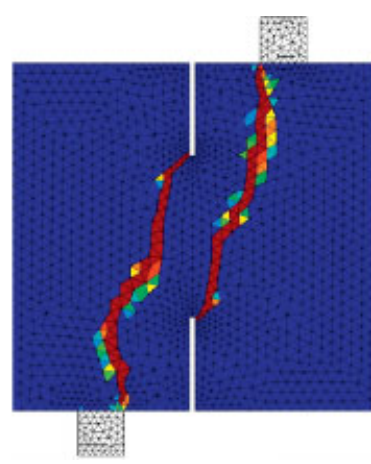

(b2)

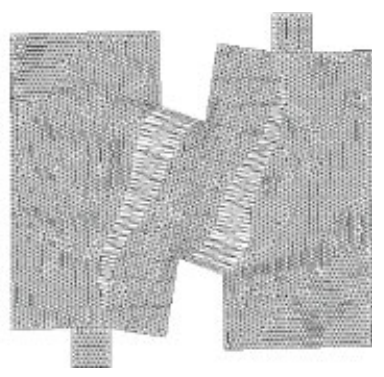

(c1)

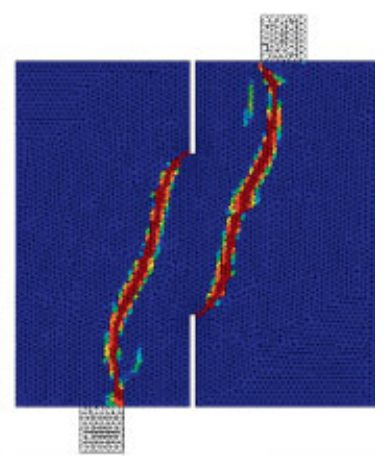

(c2)

Figure 6. Deformed geometries $(\times 100)$ and damage index contours on the three meshes with irreducible $P 1$ elements (standard Rankine criterion).

to the solution of the continuum problem and no spurious mesh bias is observed in any of the discrete solutions.

Figures 5(a.2), (b.2) and (c.2) show plots of the damage index in the three meshes. Note that in the mixed formulation damage is inter-element continuous. Results are not mesh biased in any way: the damage patterns are consistent with the three different levels of discretization.

Second, $P 1$ (linear displacement) irreducible elements are used. Figure 6 shows the results obtained, which may be compared with those in Figure 5. As it can be seen, the crack initiates correctly in all meshes, but in the three cases it turns vertically almost immediately to run along with the respective preferential mesh alignment and too close to the vertical central axis. Note, in the bottom row, that in the irreducible formulation damage is inter-element discontinuous. Results obtained, either in terms of damage distribution or strain localization, are clearly mesh biased. Therefore, convergence to the solution of the corresponding continuum problem cannot be expected.

Figure 7 shows load versus imposed vertical displacement curves obtained for the three meshes: (a) with $P 1 P 1$ mixed elements and (b) with $P 1$ irreducible elements. Curves obtained with the mixed formulation are very similar among them. Note that in the elastic regime, the three responses overlap almost exactly. The non-linear response is not spuriously affected neither by mesh size nor by mesh bias. Contrariwise, curves obtained with the irreducible formulation show trends as different among them as the corresponding localization patterns. Note that the different resolution of the meshes is evident even in the elastic regime. However, the responses, even if mesh bias dependent, are not mesh size dependent.

Third, the four-point bending beam is reanalyzed with $P 1 P 1$ mixed elements but now using the truncated Rankine damage criterion (see Equations (22b) and (23) and Figure 3(a)). The results obtained are shown in Figures 8 and 9. Again, strain localization and damage index contours are mesh bias independent. Comparing Figure 5 with Figure 8, it is obvious that the crack pattern obtained depends on the constitutive model adopted and, in this particular case, in the selection of the damage criterion. By adopting the truncated Rankine criterion, rather than the standard, the 

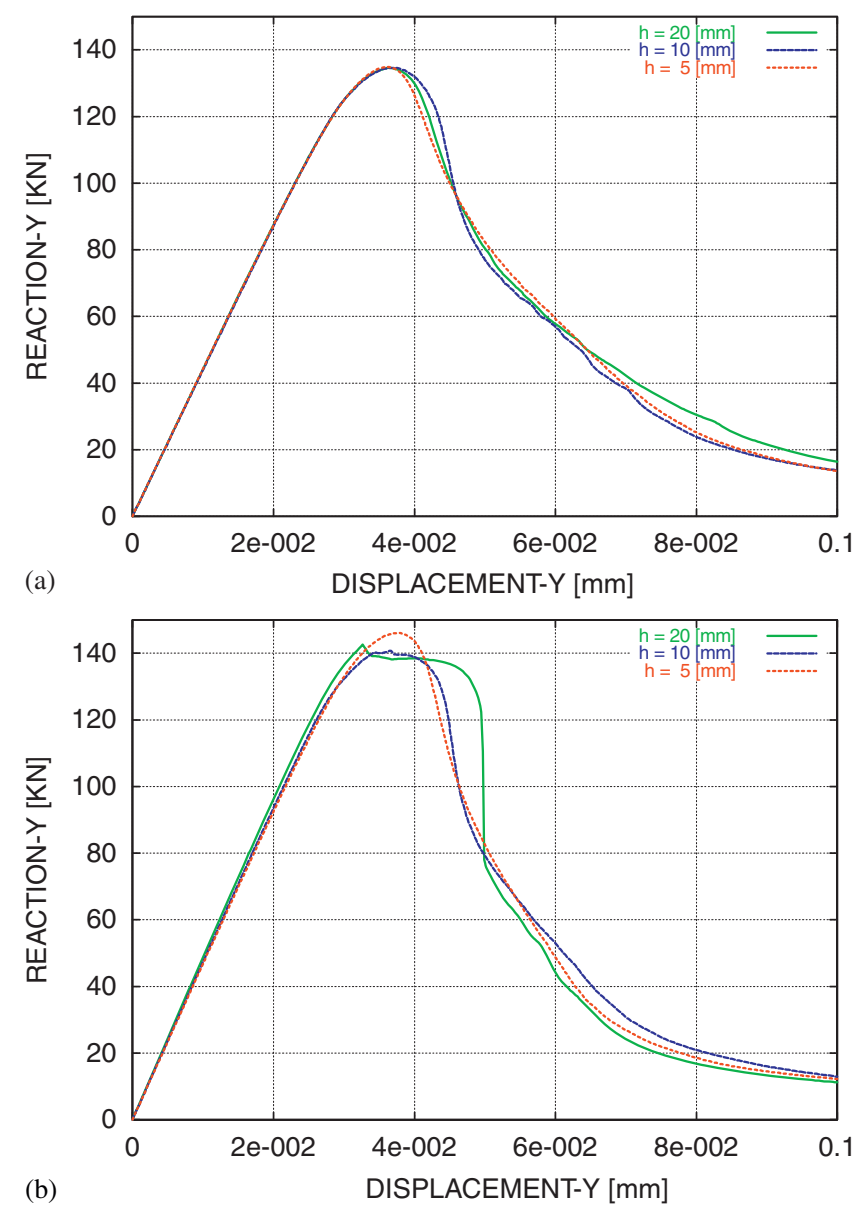

Figure 7. Load versus displacement for four-point bending beam. Standard Rankine damage criterion. Comparison between different mesh sizes: (a) $P 1 P 1$ mixed elements and (b) $P 1$ irreducible elements.

areas of the beam which are under biaxial tension are penalized in front of those closer to the compressive strut. This effect makes the crack pattern markedly more curved in the early stages. As before, the cracks turn vertically when they approach the action lines of the central reactions. Figure 9 shows the corresponding load versus imposed vertical displacement curves. As expected, the results obtained with the three meshes are consistent. Compared with the curves in Figure 7, a reduction of $30.64 \%$ in the peak load is obtained when adopting the truncated criterion; also, the development of the non-linear behavior is more sudden.

Figure 10 shows the results obtained using the stabilized mixed formulation on the fine mesh. The two columns represent the evolution, at four different time steps of the analysis, of: (a) the contours of total displacements and (b) the contours of maximum principal strain. The bottom figures show how, when the failure mechanism is fully developed, all the deformation concentrates in the formed cracks, while the elements outside these localization bands are mostly undeformed. The resolution of the cracks is optimal for the mesh used. Note in the left bottom plot how, once both cracks are formed, the central part of the beam rotates almost as a rigid body around the center of the beam. For the coarser meshes, similar results are obtained, although the strain localization is smeared across a row of larger elements (Figure 8).

Finally, Figures 11(a) and (b) show the evolution, at three different time steps of the analysis, of: (a) the horizontal displacements, (b) the maximum principal strain, along a horizontal line along the longitudinal axis of the beam which crosses both cracks. In the first one, the initially smooth gradient of displacements progressively localizes into a very sharp jump across 


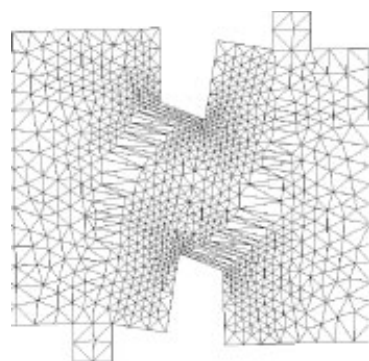

(a1)

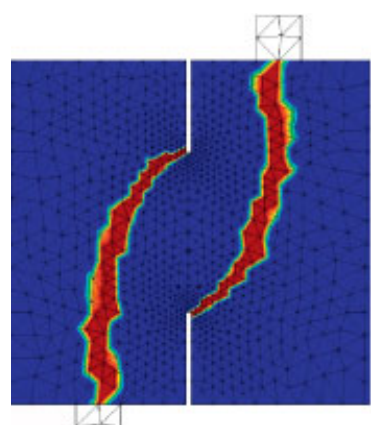

(a2)

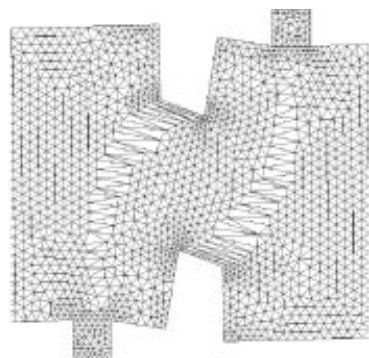

(b1)

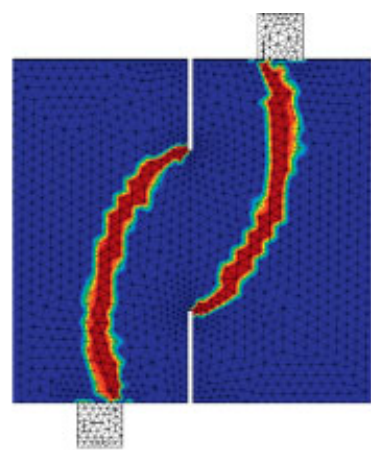

(b2)

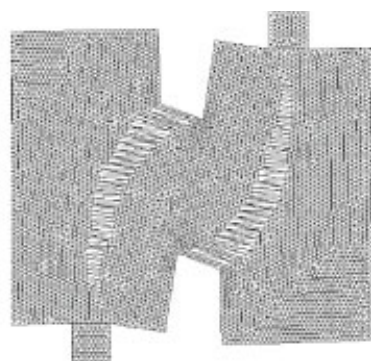

(c1)

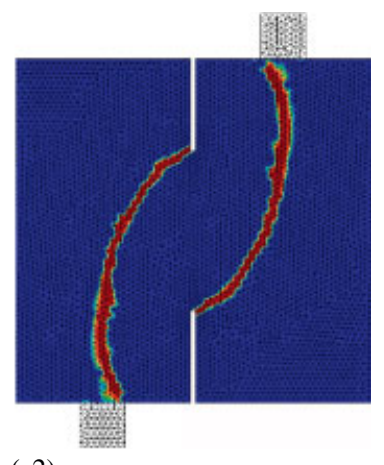

(c2)

Figure 8. Deformed geometries $(\times 100)$ and damage index contours on the three meshes with mixed $P 1 P 1$ elements (truncated Rankine criterion).

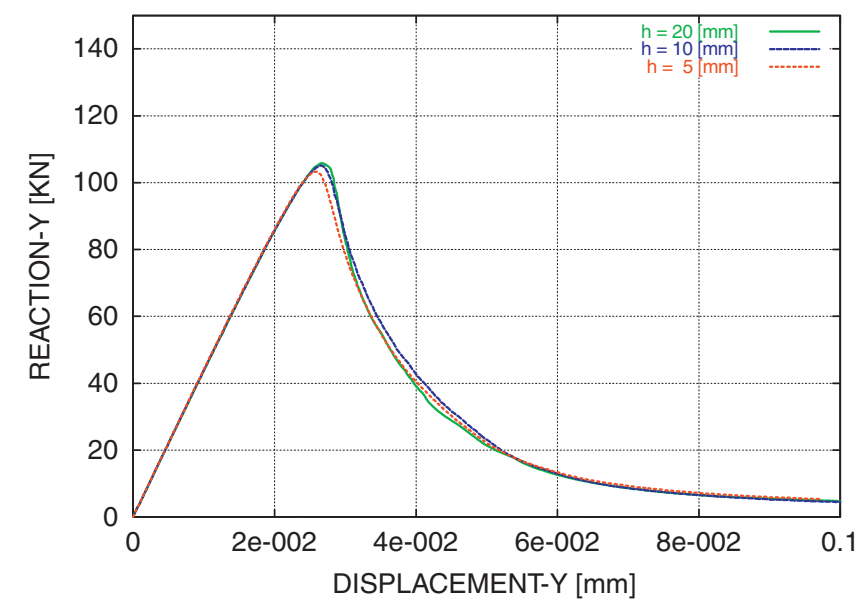

Figure 9. Load versus displacement for four-point bending beam. Truncated Rankine damage criterion. Comparison between different mesh sizes.

one single element. In the second one, the strain profile progressively localizes with very sharp resolution.

\subsection{Three-point reinforced prestressed bending beam}

The second example is a doubly notched reinforced prestressed beam subjected to three-point bending. Figure 12 depicts the geometry and dimensions of the beam, which are $167.0 \mathrm{~cm} \times 40.0 \mathrm{~cm}$ (width $\times$ height). It has a symmetric wide-flange section in which the flanges are $20.0 \mathrm{~cm} \times 5.0 \mathrm{~cm}$ and the web is $5.0 \mathrm{~cm} \times 30.0 \mathrm{~cm}$. The reinforcement consists of an unbonded platen of $10.0 \mathrm{~cm} \times$ $1.0 \mathrm{~cm}$ located in the middle of the lower flange and anchored at both ends. The two notches are 


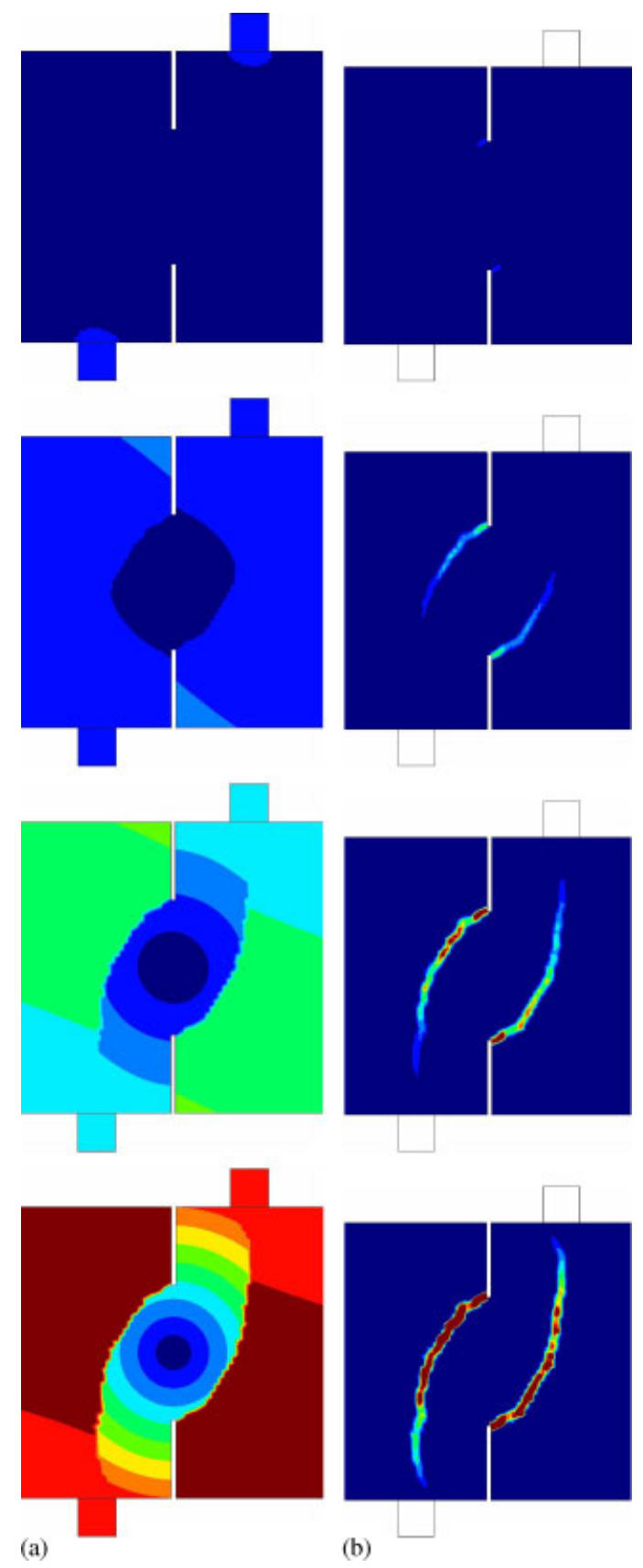

Figure 10. Results for four-point bending beam using mixed $P 1 P 1$ elements. Evolution of:

(a) displacement and (b) maximum principal strain.

symmetrically located at $40.5 \mathrm{~cm}$ from the center of the beam; the length and width of the notches are 5.0 and $1.0 \mathrm{~cm}$, respectively. The two supports near the ends of the beam (at $4.0 \mathrm{~cm}$ ) are rollers. Horizontal displacements are assumed null at the symmetry axis.

The beam is initially prestressed with two horizontal loads $H=50 \mathrm{kN}$ applied along the centers of gravity of the upper flange. After that, a central vertical point load is applied along the symmetry axis of the beam. The problem is assumed bidimensional, with different thickness for web and flanges. This example is selected because it presents a very interesting curved crack pattern.

The following material properties are assumed for the material of the web: Young's modulus $E=30 \mathrm{GPa}$, the Poisson ratio $v=0.0$, tensile strength $\sigma_{0}=3 \mathrm{MPa}$ and mode I fracture energy 

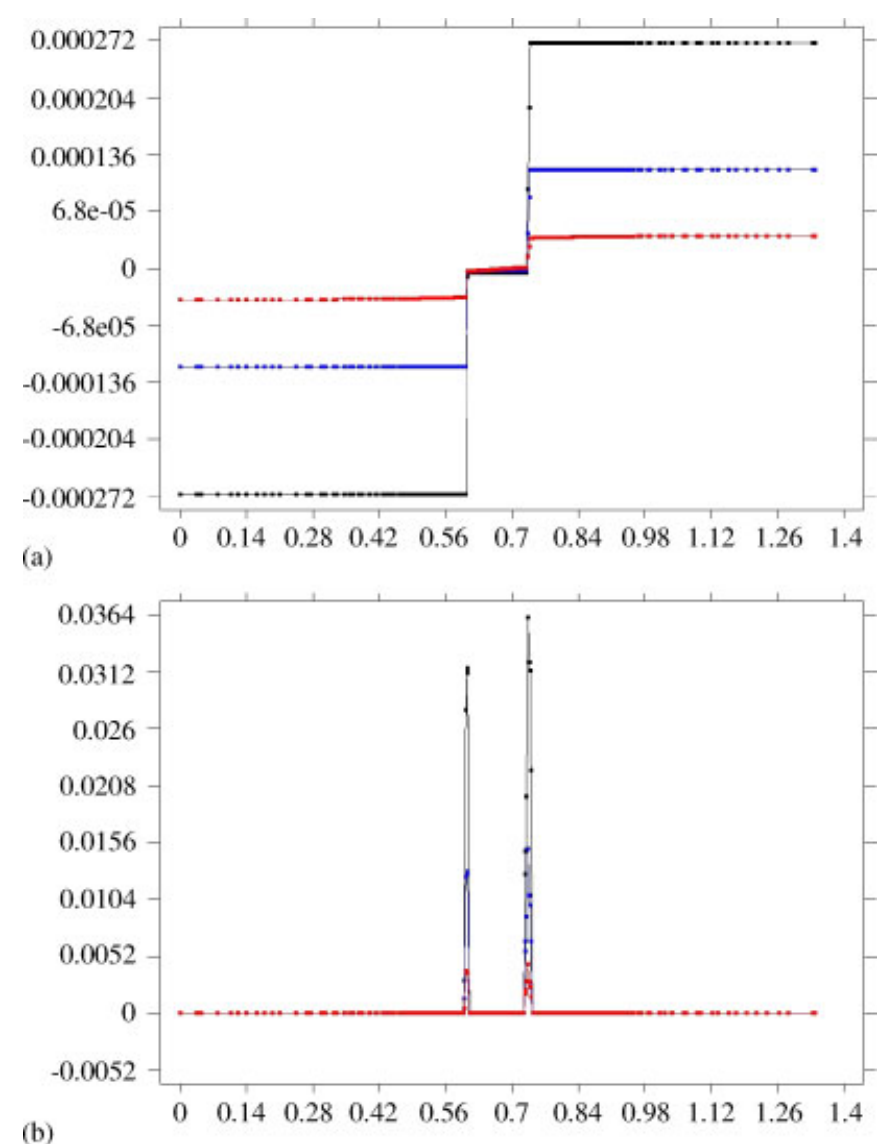

Figure 11. Evolution of the profiles along a vertical line of: (a) vertical displacement and (b) maximum principal strains for four-point bending beam.

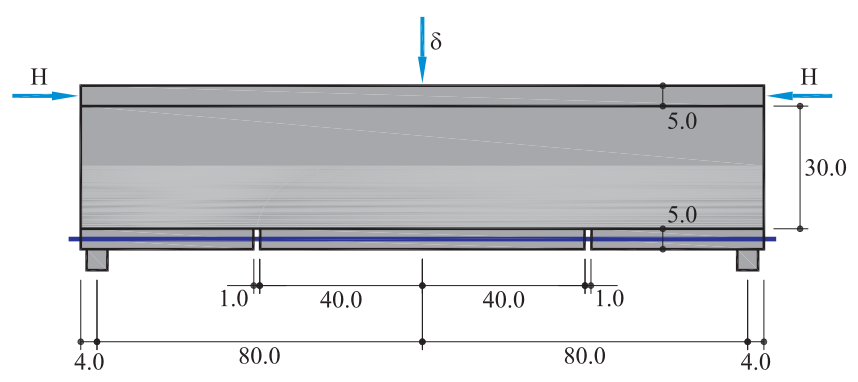

Figure 12. Three-point reinforced prestressed bending beam.

$G=25 \mathrm{~J} / \mathrm{m}^{2}$. The truncated Rankine damage criterion defined in Figure 3(a) is used, although almost identical results are obtained with the standard Rankine criterion. The flanges are assumed elastic. The reinforcement Young's modulus is $E_{\mathrm{r}}=200 \mathrm{GPa}$.

Because of symmetry, only half of the beam needs to be discretized. The right half of the beam is discretized into four different meshes of triangular elements with different mesh bias and two average mesh sizes: (a1) unstructured mesh with $h_{e}=10 \mathrm{~mm}$ (3952 nodes), (b1) unstructured mesh with $h_{e}=5 \mathrm{~mm}$ ( 8353 nodes), (a2) structured mesh with $h_{e}=10 \mathrm{~mm}$ (3651 nodes) and (b2) structured mesh with $h_{e}=5 \mathrm{~mm}$ (14180 nodes). The central part of the four meshes is shown in Figure 13; the top row corresponds to the unstructured meshes and the bottom row to the structured ones. 


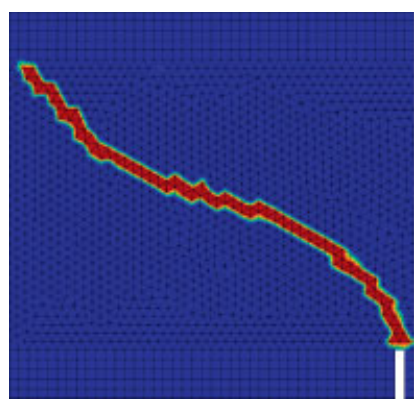

(a1)

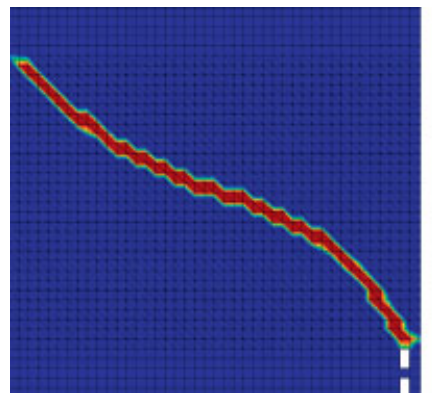

(a2)

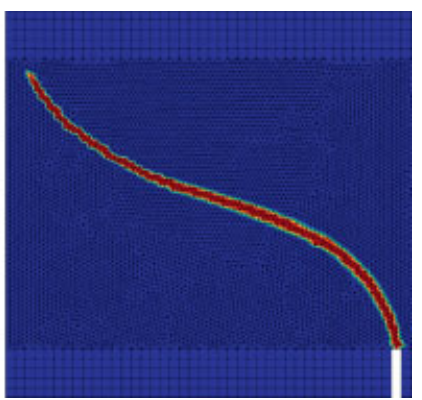

(b1)

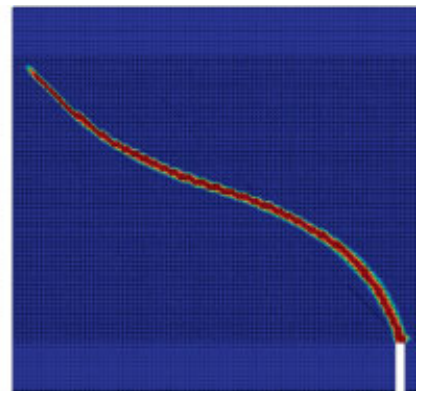

(b2)

Figure 13. Damage contours on the four meshes with mixed $P 1 P 1$ elements.

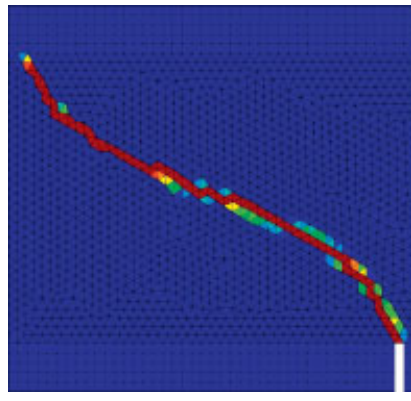

(a1)

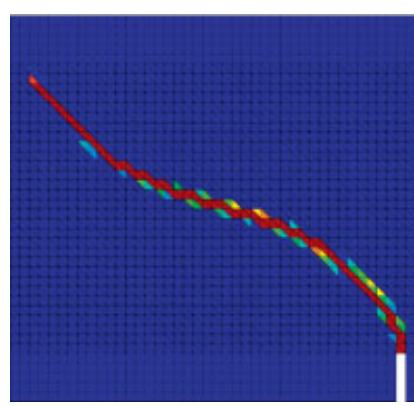

(a2)

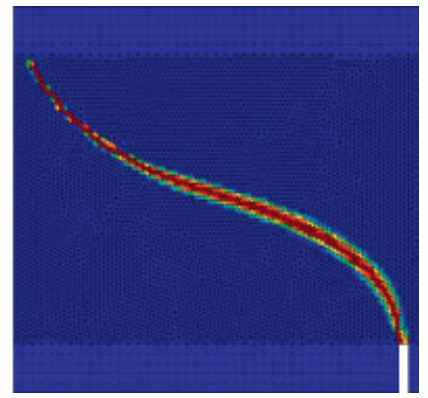

(b1)

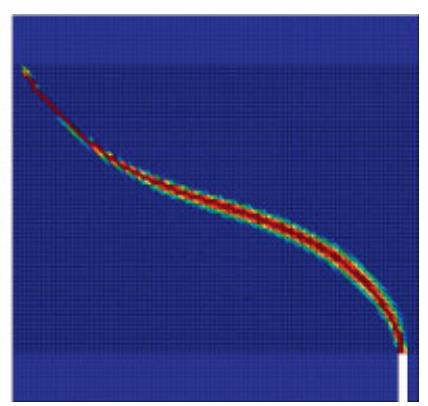

(b2)

Figure 14. Damage contours on the four meshes with irreducible $P 1$ elements.

In a first stage, $P 1 P 1$ (linear strain/linear displacement) mixed elements are used for the analysis of the beam. Figure 13 shows plots of the final damage index distribution in the four meshes considered. It is evident that the computed crack patterns in all the analyses agree very closely. The tips of the notches are singular points located at the junction of the lower flange and the 

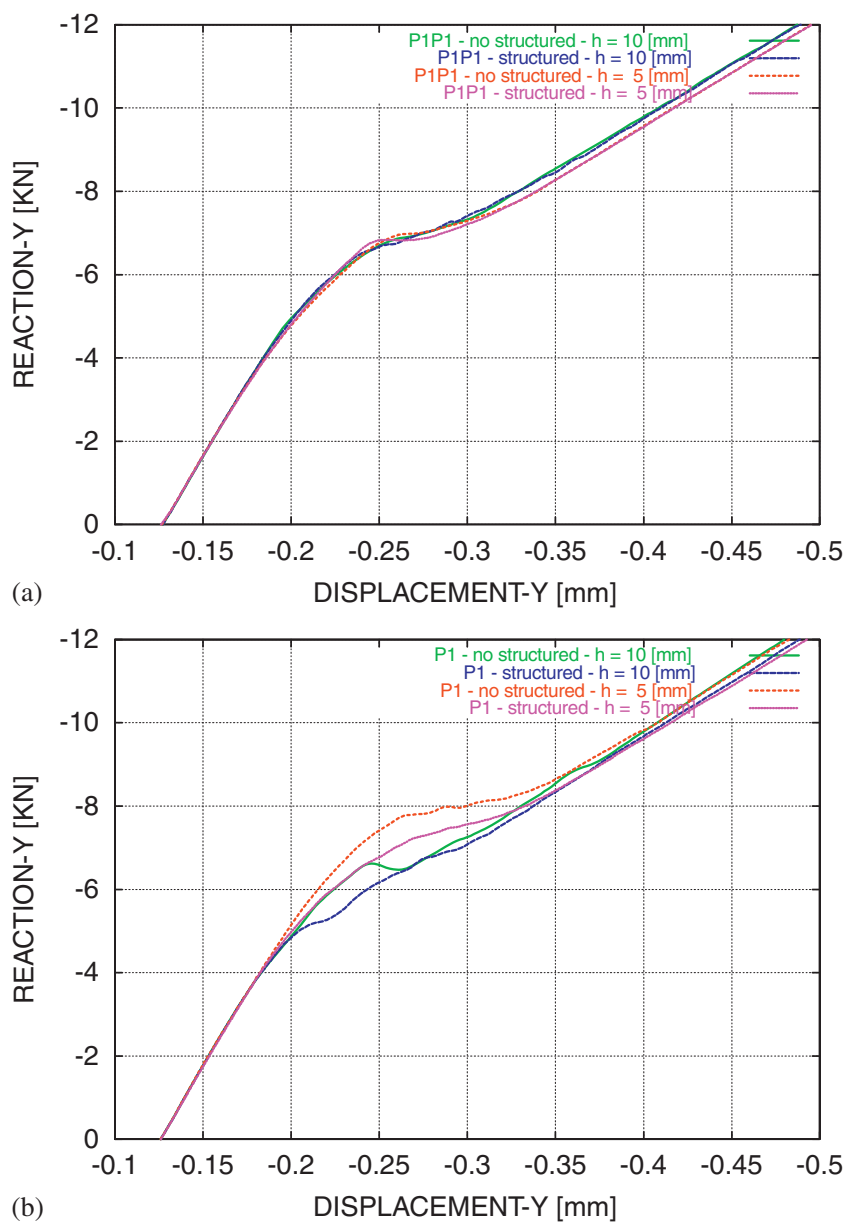

Figure 15. Load versus displacement for three-point bending beam. Comparison between different meshes: (a) $P 1 P 1$ mixed elements and (b) $P 1$ irreducible elements.

web; stresses are very large in the vicinity of these regions and damage starts there. Consequently, cracks are initiated with an inclination with respect to the horizontal axis which depends on the ratio between shear and longitudinal stresses, determined by the position of the notches along the beam. This inclination reduces as the crack progresses simultaneously toward the symmetry and neutral axes of the beam, pushing the neutral axis upwards at the same time. Finally, the cracks point toward the upper point load until they reach the junction of the web with the upper flange. The crack pattern obtained from the discrete models corresponds to the solution of the continuum problem and no spurious mesh bias is observed in any of the computed finite element solutions.

Second, the same analyses on the four meshes are repeated using $P 1$ (linear displacement) irreducible elements. The results obtained are shown in Figure 14. In this case, the crack patterns obtained do not apparently depend on the mesh alignment in a spurious manner as they are similar to those obtained with the mixed formulation, the only evident difference being that in the irreducible formulation damage is inter-element discontinuous. Unfortunately, even if not evidently incorrect, these results are not satisfactory.

This is clear in Figure 15, showing load versus imposed vertical displacement curves obtained for the four meshes: (a) with $P 1 P 1$ mixed elements and (b) with $P 1$ irreducible elements. Curves obtained with the mixed formulation are remarkably similar among them. The four curves overlap in the elastic regime. Similarly, in the final part of the non-linear response, when the cracks are fully developed, the curves pair according to their respective fineness. Therefore, the results are 

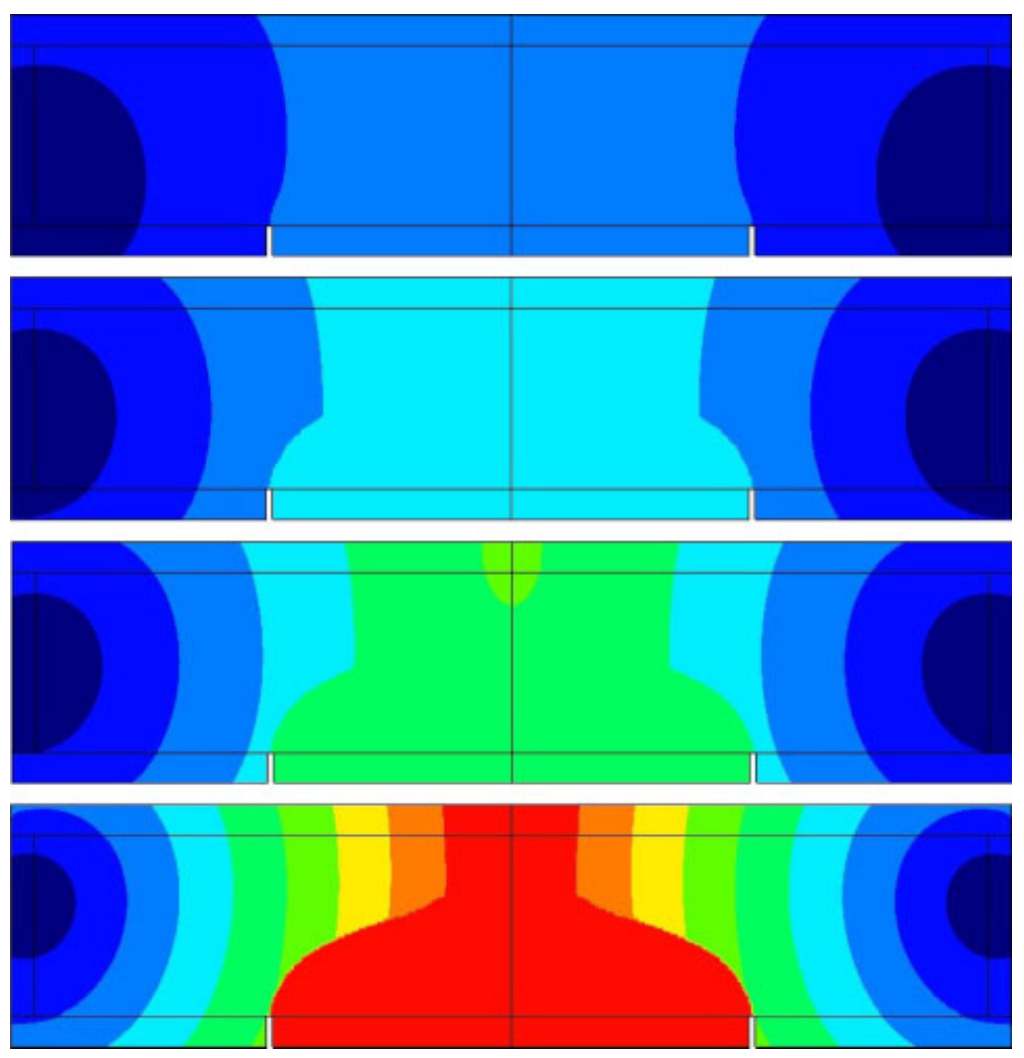

Figure 16. Evolution of displacement contours for four-point bending beam.

not spuriously affected neither by mesh size nor by mesh bias. The situation is very different for the four curves obtained with the irreducible formulation where the responses are quite evidently mesh dependent.

Figures 16 and 17 show the results obtained using the stabilized mixed formulation on the fine unstructured mesh. The two figures represent the evolution, at four different time steps of the analysis, of the contours of total displacements and the contours of the damage index, respectively. The displacement contours show how, as the cracks develop, sharp displacement gradients occur across the cracks, as it corresponds to the solution of the continuum problem. The plots also show how the neutral axis is pushed upwards by the progression of the cracks. The contours of the damage index show that the proposed formulation is capable of reproducing the formation of a collapse mechanism which depends very non-linearly on the structural response.

Finally, to demonstrate the generality of the proposed approach, the beam is analyzed using quadrilateral elements, both with $Q 1 Q 1$ (bilinear strain/bilinear displacement) mixed elements and with $Q 1$ (bilinear displacement) irreducible elements. Both meshes are structured with $h_{e}=5 \mathrm{~mm}$ (14 180 nodes). The results obtained are shown in Figure 18. On one hand, the crack pattern obtained with the mixed formulation agrees with the results obtained with the triangular linear $P 1 P 1$ elements. Nevertheless, comparing these to the results obtained for the structured mesh (b.2) in Figure 13, which has the same number of nodes in exactly the same positions, the bandwidth of the localization band is approximately doubled. It is therefore concluded that for localization problems quadrilateral elements are not as adequate as their triangular counterparts. On the other hand, the results obtained with the irreducible formulation are dependent on the mesh bias; this is obvious at the inception of the cracks, which spring vertically rather than at the correct inclination. It also shows a much increased spreading of the damage, due to the difficulty of the crack to maneuver in the structured mesh. As a result of this, the tip of the crack finally reaches the upper flange several elements off to the right from the correct point. 

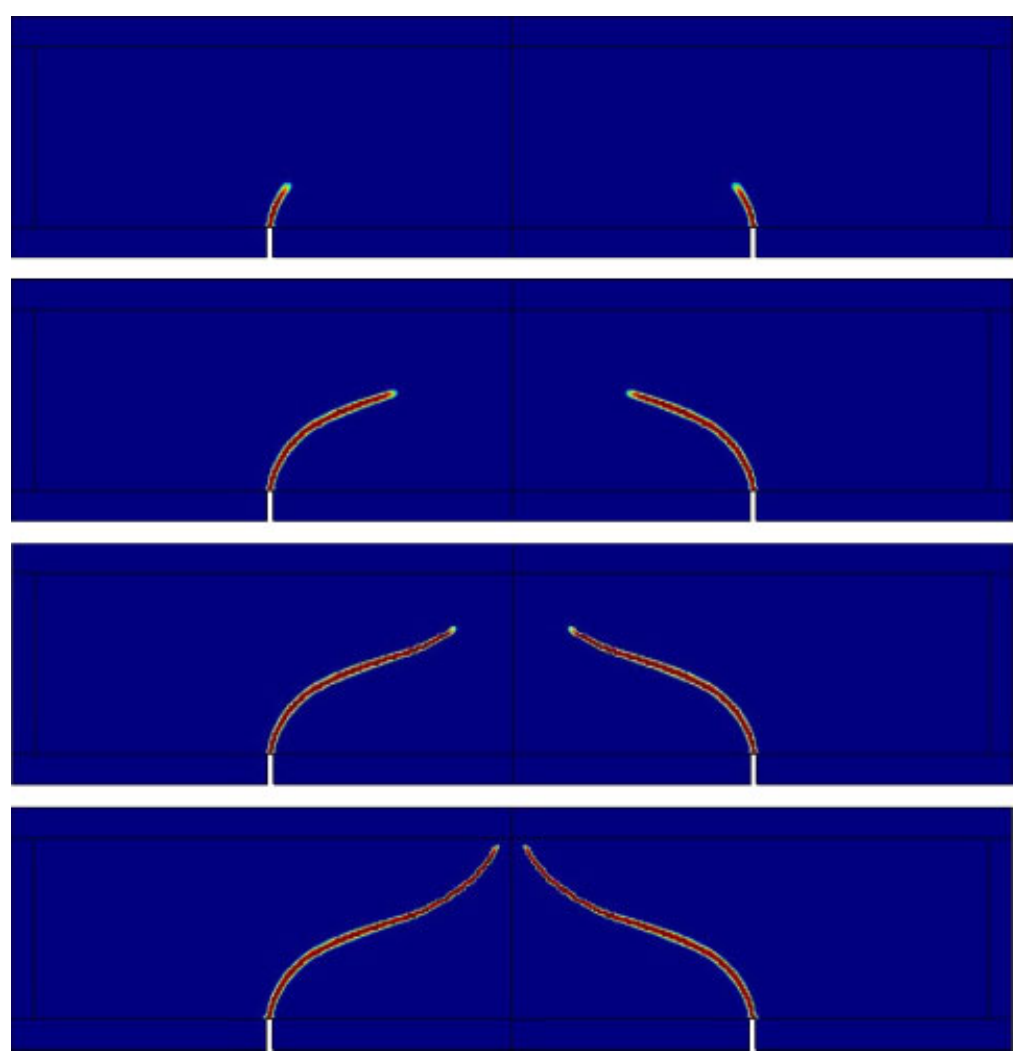

Figure 17. Evolution of damage index for three-point bending beam.

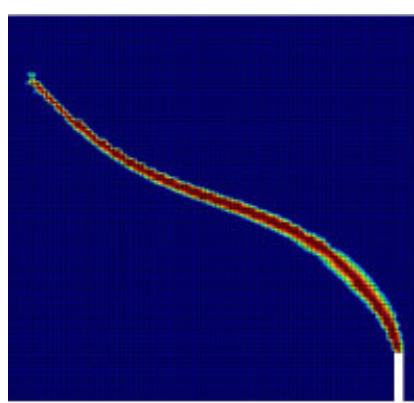

(a)

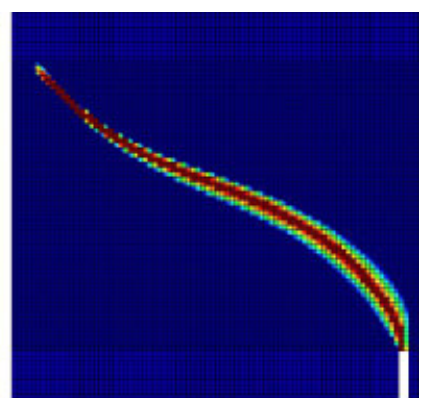

(b)

Figure 18. Damage index contours on the meshes with quadrilateral elements: (a) mixed $Q 1 Q 1$ and (b) irreducible $Q 1$.

\section{CONCLUSIONS}

This paper presents the application of a stabilized mixed equal order linear strain/displacement finite element formulation for the solution non-linear solid mechanics problems involving tensile and mixed-mode cracking. Such a formulation is adopted because the well-known observed meshbias dependence of the results obtained using the standard irreducible formulation is attributed to lack of point-wise convergence of the strains and stresses in quasi-singular situations. For the linear case, such a convergence cannot be proved for the standard approach, but it happens in the mixed case. Although rigorous analysis of the mixed formulation is not available for the non-linear case, convergence is to be expected. 
Consequently, low-order finite elements with continuous strain and displacement fields (triangular $P 1 P 1$ and quadrilateral $Q 1 Q 1$ ) and two alternative local isotropic Rankine damage models with strain-softening are used within the well-established smeared approach to crack modeling. The derived model yields a general and robust scheme, suitable for engineering applications. Its application translates in the achievement of three goals:

1. the resulting discrete FE model is well posed and stable,

2. the formulation is convergent and, on mesh refinement, it approaches the original continuum problem, and

3. the results obtained are not spuriously dependent on the finite element mesh used; they depend only on the actual material model (damage criterion in this case) adopted. No auxiliary crack tracking technique is necessary.

\section{ACKNOWLEDGEMENTS}

Financial support from the Spanish Ministry for Education and Science under the SEDUREC project (CSD2006-00060) is acknowledged.

\section{REFERENCES}

1. Ngo D, Scordelis AC. Finite element analysis of reinforced concrete beams. ACI Journal 1967; 64(14):152-163.

2. Rashid Y. Analysis of prestressed concrete pressure vessels. Nuclear Engineering and Design 1968; 7:334-344.

3. Hillerborg A, Modeer M, Peterson PE. Analysis of crack formation and crack growth in concrete by means of F. M. and finite elements. Cement and Concrete Research 1976; 6:773-782.

4. Pietruszczak S, Mróz Z. Finite element analysis of deformation of strain softening materials. International Journal for Numerical Methods in Engineering 1981; 17:327-334.

5. Bazant ZP, Oh BH. Crack band theory for fracture of concrete. Material and Structures 1983; 16:155-177.

6. Bazant ZP, Planas J. Fracture and Size Effect in Concrete and Other Quasibrittle Materials. CRC Press: Boca Raton, 1998.

7. de Borst R. Simulation of strain localization: a reppraisal of the Cosserat continuum. Engineering Computations 1991; 8:317-322.

8. Steinmann P, Willam K. Localization within the framework of micropolar elastoplasticy. In Advances in Continuum Mechanics, Mannl V et al. (eds). Springer: Berlin, 1992; 296-313.

9. de Borst R, Mulhaus HB. Gradient-dependent plasticity: formulation and algorithm aspect. International Journal for Numerical Methods in Engineering 1992; 35:521-539.

10. de Borst R, Sluys LJ, Muhlhaus HB, Pamin J. Fundamental issues in finite element analysis of localization of deformation. Engineering Computations 1993; 10:99-121.

11. Pamin J. Gradient-dependent plasticity in numerical simulation of localization phenomena. Ph.D. Thesis, TU Delft, The Netherlands, 1994.

12. Peerlings RHJ, de Borst R, Brekelmans WAM, Geers MGD. Gradient-enhanced damage modelling of concrete failures. Mechanics of Cohesive-Frictional Materials 1998; 4:339-359.

13. Gutiérrez MA, de Borst R. A unified framework for concrete damage and fracture models including size effects. International Journal of Fracture 1999; 95:261-277.

14. Simone A. Continuous-discontinuous modelling of failure. Ph.D. Thesis, TU Delft, The Netherlands, 2003.

15. Simone A, Wells GN, Sluys LJ. From continuous to discontinuous failure in a gradient-enhanced continuum damage model. Computer Methods in Applied Mechanics and Engineering 2003; 192(41-42):4581-4607.

16. Simone A, Askes H, Sluys LJ. Incorrect initiation and propagation of failure in non-local and gradient-enhanced media. International Journal of Solids and Structures 2004; 41(2):351-363.

17. Pijaudier-Cabot G, Bazant ZP. Nonlocal damage theory. Journal of Engineering Mechanics (ASCE) 1987; 113(10): $1512-1533$.

18. Pijaudier-Cabot G, Huerta A. Finite element analysis of bifurcation in nonlocal strain softening solids. Computer Methods in Applied Mechanics and Engineering 1991; 90:905-919.

19. Jirásek M. Nonlocal models for damage and fracture: comparison of approaches. International Journal of Solids and Structures 1998; 35:4133-4145.

20. Bazant Z, Jirásek M. Nonlocal integral formulations of plasticity and damage: survey of progress. Journal of Engineering Mechanics (ASCE) 2002; 128:1119-1149.

21. de Borst R. Some recent issues in computational failure mechanics. International Journal for Numerical Methods in Engineering 2001; 52:63-95.

22. Jason L, Ghavamian S, Pijaudier-Cabot G, Huerta A. Benchmarks for the validation of a non local damage model. Revue française de Génie civil 2004; 8:303-328.

23. Rodríguez-Ferran A, Morata I, Huerta A. Efficient and reliable nonlocal damage models. Computer Methods in Applied Mechanics and Engineering 2004; 193:3431-3455. 
24. Rodríguez-Ferran A, Morata I, Huerta A. A new damage model based on nonlocal displacements. International Journal for Numerical and Analytical Methods in Geomechanics 2005; 29:473-493.

25. Jirásek M, Marfia S. Nonlocal damage model based on displacement averaging. International Journal for Numerical Methods in Engineering 2005; 63:77-102.

26. Jirásek M, Rolshoven S. Localization properties of strain-softening gradient plasticity models. Part I: straingradient theories. Part II: theories with gradients of internal variables. International Journal of Solids and Structures 2009; 46:2225-2238; 2239-2254.

27. Needelman A. Material rate dependence and mesh sensitivity in localization problems. Computer Methods in Applied Mechanics and Engineering 1988; 67:68-75.

28. Wang WM, Sluys LJ, de Borst R. Viscoplasticity for instabilities due to strain softening and strain-rate softening. International Journal for Numerical Methods in Engineering 1997; 40(20):3839-3864.

29. Gutiérrez MA, de Borst R. Numerical analysis of localization using a viscoplastic regularization: influence of stochastic material defects. International Journal for Numerical Methods in Engineering 1999; 44(12):1823-1841.

30. van Zijl GPAG, de Borst R, Rots JG. A numerical model for the time-dependent cracking of cementitious materials. International Journal for Numerical Methods in Engineering 2001; 52(7):637-654.

31. Chiumenti M, Valverde Q, Agelet de Saracibar C, Cervera M. A stabilized formulation for incompressible elasticity using linear displacement and pressure interpolations. Computer Methods in Applied Mechanics and Engineering 2002; 191:5253-5264.

32. Cervera M, Chiumenti M, Valverde Q, Agelet de Saracibar C. Mixed Linear/linear simplicial elements for incompressible elasticity and plasticity. Computer Methods in Applied Mechanics and Engineering 2003; 192:5249-5263.

33. Cervera M, Chiumenti M, Agelet de Saracibar C. Softening, localization and stabilization: capture of discontinuous solutions in J2 plasticity. International Journal for Numerical and Analytical Methods in Geomechanics 2003; 28:373-393.

34. Cervera M, Chiumenti M, Agelet de Saracibar C. Shear band localization via local $J_{2}$ continuum damage mechanics. Computer Methods in Applied Mechanics and Engineering 2003; 193:849-880.

35. Chiumenti M, Valverde Q, Agelet de Saracibar C, Cervera M. A stabilized formulation for incompressible plasticity using linear triangles and tetrahedra. International Journal of Plasticity 2004; 20:1487-1504.

36. Agelet de Saracibar C, Chiumenti M, Valverde Q, Cervera M. On the orthogonal subgrid scale pressure stabilization of finite deformation $J_{2}$ plasticity. Computer Methods in Applied Mechanics and Engineering 2006; 195:1224-1251.

37. Cervera M, Chiumenti M. Size effect and localization in $J_{2}$ plasticity. International Journal of Solids and Structures 2009; 46:3301-3312.

38. Cervera M, Chiumenti M, Codina R. Mixed stabilized finite element methods in nonlinear solid mechanics. Part I: formulation. Computer Methods in Applied Mechanics and Engineering 2010; 199:2559-2570.

39. Cervera M, Chiumenti M, Codina R. Mixed stabilized finite element methods in nonlinear solid mechanics. Part II: strain localization. Computer Methods in Applied Mechanics and Engineering 2010; 199:2571-2589.

40. Djoko JK, Lamichhane BP, Reddy BD, Wohlmuth BI. Conditions for equivalence between the Hu-Washizu and related formulations, and computational behavior in the incompressible limit. Computer Methods in Applied Mechanics Engineering 2006; 195:4161-4178.

41. Badia S, Codina R. Unified stabilized finite element formulations for the Stokes and the Darcy problems. SIAM Journal on Numerical Analysis 2009; 17:309-330.

42. Badia S, Codina R. Stabilized continuous and discontinuous Galerkin techniques for Darcy flow. Computer Methods in Applied Mechanics and Engineering 2010; 199:1654-1667.

43. Brezzi F, Fortin M. Mixed and Hybrid Finite Element Methods. Springer: New York, 1991.

44. Mijuca D. On hexahedral finite element HC8/27 in elasticity. Computational Mechanics 2004; 33:466-480.

45. Hughes TJR. Multiscale phenomena: Green's function, Dirichlet-to Neumann formulation, subgrid scale models, bubbles and the origins of stabilized formulations. Computer Methods in Applied Mechanics and Engineering 1995; 127:387-401.

46. Codina R. Stabilized finite element approximation of transient incompressible flows using orthogonal subscales. Computer Methods in Applied Mechanics and Engineering 2002; 191:4295-4321.

47. Codina R, Principe J. Dynamic subscales in the finite element approximation of thermally coupled incompressible flows. International Journal for Numerical Methods in Fluids 2007; 54:707-730.

48. Hughes TJR, Feijoó GR, Mazzei L, Quincy JB. The variational multiscale method—a paradigm for computational mechanics. Computer Methods in Applied Mechanics and Engineering 1998; 166:3-28.

49. de Borst R. Isotropic and anisotropic damage models for concrete fracture. In Mechanics of Quasi-Brittle Materials and Structures, Pijaudier-Cabot G et al. (eds). Hermes Sci. Pubs.: London, 1999; 39-56.

50. de Borst R. Fracture in quasi-brittle materials: a review of continuum damage-based approaches. Engineering Fracture Mechanics 2002; 69:95-112.

51. Cervera M, Hinton E, Hassan O. Non linear analysis of reinforced concrete plate and shell structures using 20-noded isoparametric brick elements. Computers and Structures 1987; 25:845-869.

52. Cervera M, Chiumenti M. Smeared crack approach: back to the original crack. International Journal for Numerical and Analytical Methods in Geomechanics 2006; 30:1173-1199.

53. Cervera M, Chiumenti M. Mesh objective tensile cracking via a local continuum damage model and a crack tracking technique. Computer Methods in Applied Mechanics and Engineering 2006; 196(1-3):304-320. 
54. Cervera M. An orthotropic mesh corrected crack model. Computer Methods in Applied Mechanics and Engineering 2008; 197:1603-1619.

55. Cervera M. A smeared-embedded mesh-corrected damage model for tensile cracking. International Journal for Numerical Methods in Engineering 2008; 76:1930-1954.

56. Oliver J. A consistent characteristic length for smeared cracking models. International Journal for Numerical Methods in Engineering 1989; 28:461-474.

57. Cervera M, Agelet de Saracibar C, Chiumenti M. COMET: COupled MEchanical and Thermal analysis. Data Input Manual, Version 5.0, Technical Report IT-308, 2002. Available from: http://www.cimne.upc.es.

58. GiD: The Personal Pre and Post Processor, 2009. Available from: http://www.gidhome.com. 